\title{
SYMMETRIC POSITIVE SYSTEMS WITH BOUNDARY CHARACTERISTIC OF CONSTANT MULTIPLICITY
}

\author{
BY
}

\author{
JEFFREY RAUCH ${ }^{1}$
}

\begin{abstract}
ABSTRACr. The theory of maximal positive boundary value problems for symmetric positive systems is developed assuming that the boundary is characteristic of constant multiplicity. No such hypothesis is needed on a neighborhood of the boundary. Both regularity theorems and mixed initial boundary value problems are discussed. Many classical ideas are sharpened in the process.
\end{abstract}

1. Introduction. Suppose that $\Omega \subset \mathbf{R}^{\nu}$ is a bounded open set lying on one side of its $C^{1}$ boundary $\partial \Omega$. In $\bar{\Omega}$ suppose that

$$
L=\sum_{j=1}^{\nu} A_{j}(x) \partial_{j}+B(x)
$$

is a first order system of differential operators with

$$
\begin{gathered}
A_{j} \in \operatorname{Lip}\left(\bar{\Omega}: \operatorname{Hom}\left(\mathbf{C}^{k}\right)\right), \\
B \in L^{\infty}\left(\bar{\Omega}: \operatorname{Hom}\left(\mathbf{C}^{k}\right)\right) .
\end{gathered}
$$

We are interested in boundary value problems for the system

$$
L u=f \in \mathscr{L}^{2}(\Omega) .
$$

Our first result is concerned with Green's identity,

$$
\int_{\Omega}\langle L u, v\rangle=\int_{\Omega}\left\langle u, L^{*} v\right\rangle+\int_{\partial \Omega}\left\langle A_{n} u, v\right\rangle d \sigma
$$

when $L^{*}$ is the formal adjoint of $L, n=\left(n_{1}, n_{2}, \ldots, n_{\nu}\right)$ is the unit outward normal to $\partial \Omega$ and $A_{n} \equiv \sum_{j} n_{j} A_{j}$. It has long been recognized that if $u \in \mathscr{L}^{2}(\Omega)$ and $L u \in \mathscr{L}^{2}(\Omega)$, then $\left.A_{n} u\right|_{\partial \Omega} \in H^{-1 / 2}(\partial \Omega)$ and Green's identity holds for $v \in H^{1}(\Omega)$. In fact, less is needed. Let

$$
\begin{array}{ll}
\mathscr{K}_{L}=\left\{u \in \mathscr{L}^{2}(\Omega) \mid L u \in H^{1}(\Omega)^{\prime}\right\}, & \|u\|_{\mathscr{X}_{L}}^{2}=\|u\|_{\mathscr{L}^{2}(\Omega)}^{2}+\|L u\|_{H^{1}(\Omega)^{\prime}}^{2}, \\
\mathscr{H}_{L}=\left\{u \in \mathscr{L}^{2}(\Omega) \mid L u \in \mathscr{L}^{2}(\Omega)\right\}, & \|u\|_{\mathscr{H}_{L}}^{2}=\|u\|_{\mathscr{L}^{2}(\Omega)}^{2}+\|L u\|_{\mathscr{L}^{2}(\Omega) .}^{2}
\end{array}
$$

The space $\mathscr{H}_{L^{*}}$ is defined similarly.

Proposition 1. $\mathscr{K}_{L}$ and $\mathscr{H}_{L}$ are Hilbert spaces and $C^{1}(\bar{\Omega})$ is dense in each of them.

Received by the editors June 20,1984 .

1980 Mathematics Subject Classification. Primary 35M05, 35L50, 35B30, 35A05.

Key words and phrases. Symmetric positive boundary value problem, characteristic boundary, dissipative boundary conditions, mixed initial boundary value problem.

${ }^{1}$ Partially supported by NSF Grant MCS 8301061 . 
The proof of this and other results is given in later sections. If $u \in \mathscr{L}^{2}(\Omega)$, then automatically $L u \in \stackrel{\circ}{H}^{1}(\Omega)^{\prime}$ so $\mathscr{K}_{L}$ is only slightly smaller than $\mathscr{L}^{2}(\Omega)$, the restriction coming near $\partial \Omega$.

\section{THEOREM 1. The map}

$$
\left.C^{1}(\bar{\Omega}) \ni u \mapsto A_{n} u\right|_{\partial \Omega}
$$

extends uniquely to a continuous linear map $\mathscr{K}_{L} \rightarrow H^{-1 / 2}(\partial \Omega)$ and Green's identity (5) holds for $u \in \mathscr{K}_{L}, v \in H^{1}(\Omega)$.

In the proofs of energy inequalities one wants to take $u=v$ and for that purpose this theorem is not sufficient. For $X \subset \mathbf{R}^{\nu}$ we denote by $\operatorname{Lip}(X)$ the set of uniformly Lipshitzean functions on $X$ normed by

$$
\|u\|_{\operatorname{Lip}(X)}=\sup _{x \in X}\|u(x)\|+\sup _{\substack{x, y \in X \times X \\ x \neq y}} \frac{\|u(x)-u(y)\|}{\|x-y\|} .
$$

THEOREM 2. The map

$$
C^{1}(\bar{\Omega}) \times\left. C^{1}(\bar{\Omega}) \ni(u, v) \mapsto\left\langle A_{n} u, v\right\rangle\right|_{\partial \Omega}
$$

extends uniquely to a continuous bilinear map $\mathscr{H}_{L} \times \mathscr{H}_{L^{*}} \rightarrow \operatorname{Lip}(\partial \Omega)^{\prime}$ and Green's identity (5) holds for $(u, v) \in \mathscr{H}_{L} \times \mathscr{H}_{L^{*}}$.

REMARK. The space $\operatorname{Lip}(\partial \Omega)^{\prime} \subset \mathscr{D}^{\prime}(\partial \Omega)$ does not have a useful elementary description.

REMARK. The boundary integral in Green's identity is interpreted as the action of $\left\langle A_{n} u, v\right\rangle$ on the Lipshitz continuous function 1 .

The next result expresses the idea that traces on nearby surfaces are close. Note that $u \in \mathscr{H}_{L}$ (resp. $u \in \mathscr{K}_{L}$ ) implies that $\phi u \in \mathscr{H}_{L}$ (resp. $\mathscr{K}_{L}$ ) for $\phi \in C^{1}(\bar{\Omega})$. Thus it suffices to consider functions supported in a small neighborhood of a point $p \in \partial \Omega$. Introduce local coordinates $\left(x_{1}, x^{\prime}\right)$ near $p$ so that $\Omega$ becomes $\left\{|x|<1\right.$ and $\left.x_{1}>0\right\}$. Theorem 2 then implies that for $\varepsilon \geqslant 0$, and $u \in \mathscr{K}_{L},\left.A_{1} u\right|_{x_{1}=s} \in H^{-1 / 2}\left(\mathbf{R}^{\nu-1}\right) \cap$ $\mathscr{E}^{\prime}\left(\mathbf{R}^{\nu-1}\right)$, where $A_{1}$ comes from the expression for $L$ in the new coordinates.

THEOREM 3. If $u \in \mathscr{K}_{L}$ is supported in the coordinate patch above, then the map

$$
\left.\overline{\mathbf{R}}_{+} \ni s \mapsto A_{1} u\right|_{x_{1}=s} \in H^{-1 / 2}\left(\mathbf{R}^{\nu-1}\right)
$$

is continuous. Similarly if $u \in \mathscr{H}_{L}, v \in \mathscr{H}_{L^{*}}$ are supported in the patch, then the map

$$
\left.\overline{\mathbf{R}}_{+} \ni s \mapsto\left\langle A_{1} u, v\right\rangle\right|_{x_{1}=s} \in \operatorname{Lip}\left(\mathbf{R}^{\nu-1}\right)^{\prime}
$$

is continuous.

We are interested in boundary value problems for the system (4). For simplicity we consider homogeneous linear conditions $u(x) \in N(x)$ for $x \in \partial \Omega$ when $N(x)$ is a linear subspace of $\mathbf{C}^{k}$ for each $x \in \partial \Omega$. We suppose that

$N(x)$ depends Lipshitz continuously on $x$,

$$
N(x) \supset \operatorname{ker} A_{n}(x) \text { for all } x \in \partial \Omega .
$$


Roughly speaking, since $A_{n}(x) u(x)$ is meaningful in $x \in \partial \Omega$ we expect that $u(x)$ is determined modulo $\operatorname{ker} A_{n}(x)$ and since $N(x) \supset \operatorname{ker} A_{n}(x)$ the equivalence class $u(x) \bmod N(x)$ is determined. To make this precise let $\pi_{N}(x): \mathbf{C}^{k} \rightarrow \mathbf{C}^{k} / N(x)$ be the canonical projection. Since $N(x) \supset \operatorname{ker} A_{n}(x)$ there is a unique $M(x)$ so that

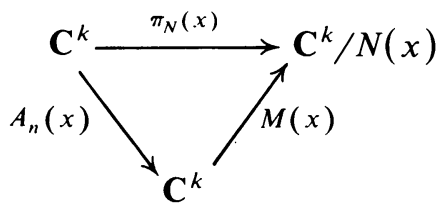

is a commutative diagram. Since $N(x)$ and $M(x)$ are Lipshitzean, $\mathbf{C}^{k} / N(x)$ is a Lipshitz continuous vector bundle over $\partial \Omega$ and $M$ is a Lipshitzean bundle map. The Sobolev space of sections $H^{s}\left(\partial \Omega: \mathbf{C}^{k} / N(x)\right)$ is well defined for $|s| \leqslant 1$. These remarks and Proposition 1 yield the following result.

Proposition 2. The map

$$
C^{1}(\bar{\Omega}) \ni u \mapsto u \bmod N(x) \in \operatorname{Lip}\left(\partial \Omega: \mathbf{C}^{k} / N(x)\right)
$$

extends uniquely to a continuous map from $\mathscr{K}_{L}$ to $H^{-1 / 2}\left(\partial \Omega: \mathbf{C}^{k} / N(x)\right)$. For $u$ in $\mathscr{K}_{L}$ the image is equal to $M\left(\left.A_{n} u\right|_{\partial \Omega}\right)$.

Definition 1. For $u \in \mathscr{K}_{L}$ we say that $u \in N$ at $\partial \Omega$ if the image of $u$ in

$$
H^{-1 / 2}\left(\partial \Omega: \mathbf{C}^{k} / N(x)\right)
$$

vanishes.

Inhomogeneous boundary conditions, $u \equiv g \bmod N$ at $\partial \Omega$, can be reduced to the homogeneous case when $g \in H^{1 / 2}(\partial \Omega)$ by merely subtracting an element of $H^{1}$ which achieves these boundary data. The adjoint boundary space $N^{*}(x)$ is defined by

$$
N^{*}(x) \equiv\left[A_{n}(x)(N(x))\right]^{\perp} .
$$

Since $N(x) \supset \operatorname{ker} A_{n}(x)$ we see that

$$
\operatorname{dim} A_{n}(N)=\operatorname{dim} N(x)-\operatorname{dim} \operatorname{ker} A_{n}(x) .
$$

Thus $N^{*}$ has locally constant dimension if and only if the nullity of $A_{n}(x)$ is locally constant.

Definition 2. The boundary of $\Omega$ is characteristic of constant multiplicity if $\operatorname{dim} \operatorname{ker} A_{n}(x)$ is constant on each component of $\partial \Omega$.

In this case, $N^{*}$ is Lipshitz continuous. We will assume from here on that $\partial \Omega$ is characteristic of constant multiplicity. The next result is fundamental.

THEOREM 4 (WEAK $=$ STRONG). If $u \in \mathscr{K}_{L}\left(\right.$ resp. $\left.\mathscr{H}_{L}\right)$ and $u \in N$ at $\partial \Omega$, then there is a sequence $u_{k} \in C^{1}(\bar{\Omega})$ with $u_{k}(x) \in N(x)$ for $x \in \partial \Omega$ and $u_{k} \rightarrow u$ in $\mathscr{K}_{L}$ (resp. $\mathscr{H}_{L}$ ).

As a consequence, it is not difficult to prove. 
Proposition 3. If $\partial \Omega$ is characteristic of constant multiplicity, $u \in \mathscr{K}_{L}$ and $f=L u$, then $u \in N$ at $\partial \Omega$ if and only if for all $v \in \operatorname{Lip}(\bar{\Omega})$ with $v(x) \in N^{*}(x)$ for all $x \in \partial \Omega$,

$$
\int_{\Omega}\left\langle u, L^{*} v\right\rangle=f(v) \text {. }
$$

Here the right-hand side is the value of $f \in H^{1}(\Omega)^{\prime}$ at $v$.

REMARK. In case $f \in \mathscr{L}^{2}(\Omega)$, the right-hand side is an integral and the formula (8) was used by Friedrichs as the definition of a weak solution to the boundary value problem.

ReMark. Using Theorem 2 we see that for $u \in \mathscr{H}_{L}$, the equality (8) extends to all $v \in \mathscr{H}_{L}$ satisfying $v \in N^{*}$ at $\partial \Omega$ in the sense of Definition 1.

Theorems 1-4 provide the basic calculus on which the theory of boundary value problems for (4) is built. Most earlier work on the subject assumed a stronger hypothesis than in Definition 2. They assumed that there was an extension of $n(x)$ to a $C^{1}$ function on $\bar{\Omega}$ so that $\operatorname{dim} \operatorname{ker} A_{n}(x)$ was constant on a neighborhood of each component of the boundary. In problems involving the flow of fluids it is quite common for this stronger hypothesis to fail (see $[1,3,13,22]$ ). This paper was written to provide a theory which was sufficiently strong to handle these problems and, secondly, refines and simplifies the standard results, even when the stronger hypothesis is valid. Another class of problems arises when $\operatorname{dim} \operatorname{ker} A_{n}(x)$ is not locally constant on $\partial \Omega$. Here examples are known when weak is not equal to strong (see $[12,14,17])$. Some positive results can be found in $[17,20]$.

The symmetric positive problems we study have an elementary a priori estimate thanks to two positivity assumptions. First we suppose that $L$ is symmetric positive, that is $A_{j}=A_{j}^{*}$ for all $x \in \Omega$, and there is a constant $a>0$ so that

$$
Z(x) \equiv \frac{B+B^{*}}{2}-\sum_{j} \partial_{j} A_{j} \geqslant a I
$$

for all $x \in \Omega$. Second, we suppose that $N$ is maximal positive in the sense that

$$
\left\langle A_{n}(x) v, v\right\rangle \geqslant 0 \quad \forall x \in \partial \Omega, v \in N(x) .
$$

$$
\operatorname{dim} N=\# \text { nonnegative eigenvalues of } A_{n} \text { counting multiplicity. }
$$

The maximality condition (11) implies that $N$ cannot be enlarged while preserving (10), in particular it implies that $N \supset \operatorname{ker} A_{n}$. If $u \in H^{1}(\Omega)$, then Green's identity (5) with $u=v$ yields the energy identity

$$
\operatorname{Re}(u, f)_{\Omega}=(Z u, u)_{\Omega}+\int_{\partial \Omega}\left\langle A_{n} u, u\right\rangle d \sigma .
$$

The positivity hypotheses (9) and (10) yield the $L^{2}$ a priori estimate

$$
a\|u\|_{\mathscr{L}^{2}(\Omega)} \leqslant\|L u\|_{\mathscr{L}^{2}(\Omega)}
$$

for $u \in H^{1}(\Omega)$ with $u(x) \in N(x)$ for almost all $x \in \partial \Omega$. Using Theorems 2 and 4 it is easy to prove the following.

THEOREM 5. For any $f \in \mathscr{L}^{2}(\Omega)$ there is a unique $u \in \mathscr{L}^{2}(\Omega)$ satisfying $L u=f$ in $\Omega$ and $u \in N$ at $\partial \Omega$. In addition the distribution $\left.\left\langle A_{n} u, u\right\rangle\right|_{\partial \Omega}$ is nonnegative, and the estimate (13) holds. 
REMARK. For $A_{n}$ invertible this was proved by Friedrichs [6] and a direct proof valid under the more restrictive constant multiplicity hypothesis was given by Lax and Phillips [9].

For problems with characteristic boundary, one does not expect full regularity of $u$ even if $f \in C^{\infty}(\bar{\Omega})$. However, there is a good tangential regularity theorem.

Definition 3. A smooth vector field $\gamma$ on $\bar{\Omega}$ is called tangential if and only if, for every $x \in \partial \Omega,\langle\gamma(x), n(x)\rangle=0$. For $s \in \mathbf{Z}_{+}$, the space $H_{\tan }^{s}(\Omega)$ consists of those $u \in \mathscr{L}^{2}(\Omega)$ with the property that for any $l \leqslant s$ and tangential fields $\left\{\gamma_{i}\right\}_{i=1}^{l}$, $\gamma_{1} \gamma_{2} \cdots \gamma_{l} u \in \mathscr{L}^{2}(\Omega)$. Clearly elements of $H_{\text {tan }}^{s}$ lie in $H_{\mathrm{loc}}^{s}(\Omega)$. Near $p \in \partial \Omega$ one may localize to $\phi u$ then introduce coordinates $\left(x_{1}, x^{\prime}\right)$ so that supp $\phi u \subset\{|x|<1$ and $\left.x_{1} \geqslant 0\right\}$. The elements $\phi u$ are characterized by

$$
\sum_{|\alpha| \leqslant s}\left\|\left(x_{1} \partial_{1}, \partial_{2}, \ldots, \partial_{\nu}\right)^{\alpha} \phi u\right\|_{\mathscr{L}^{2}\left(\mathbf{R}_{+}^{\nu}\right)}^{2}<\infty .
$$

This yields a natural Hilbert space structure for $H_{\tan }^{s}(\Omega)$ (see [2]). Assuming that $\partial \Omega$ and the coefficients of $L$ are sufficiently regular, one has tangental regularity as follows.

TheOREM 6. Suppose $s \in \mathbf{Z}_{+}, A, N$ and $\partial \Omega$ are of class $C^{s, 1}$ and $B$ is of class $C^{s-1,1}$. Then there are real numbers $\lambda_{s}$ and $C_{s}$ so that $\lambda_{0} \leqslant \lambda_{1} \leqslant \cdots$ and if $u \in H_{\tan }^{s}, u \in N$ at $\partial \Omega$, and $L u \in H_{\text {tan }}^{s}$, then for all $\lambda \in \mathrm{C}$

$$
\operatorname{Re}\left(\lambda-\lambda_{s}\right)\|u\|_{H_{\mathrm{tan}}^{s}} \leqslant C_{s}\left(\|(L+\lambda) u\|_{H_{\mathrm{tan}}^{s}}+|\lambda|\|u\|_{H_{\mathrm{tan}}^{s-1}}\right) .
$$

Conversely if $\lambda_{s}, C_{s}$ are as above, $\operatorname{Re} \lambda>\lambda_{s}$ and $f \in H_{\tan }^{s}(\Omega)$, then the unique solution $u$ to $(L+\lambda) u=f, u \in N$ at $\partial \Omega$, lies in $H_{\tan }^{s}(\Omega)$.

REMARK 1. In case $\partial \Omega$ is noncharacteristic it follows that $u \in H^{s}(\Omega)$. In the characteristic case, one cannot expect full regularity even if $f \in H^{s}(\Omega)$ (see $[11,24]$ ). However, for some important problems of mathematical physics one does get full regularity (see [11, 13, 22]).

REMARK 2. An example of Friedrichs [6] shows that without a condition that $\lambda$ be sufficiently large ( $\lambda>0$ does not always suffice) one gets regularity no better than $\mathscr{L}^{2}$.

REMARK 3. In the noncharacteristic case with $s=1$ this result was proved by Friedrichs. Higher $s$ was studied by many authors [7, 15, 16, 23]. Problems characteristic of constant multiplicity on a neighborhood of the boundary were studied in [11, 16, 24], where partial results can be found.

Results analogous to Theorems 1-6 are valid for time dependent problems $\left(\partial_{t}-L(t)\right) u=f$ in cylindrical domains $[0, T] \times \Omega$. These results are described and proved in $\S 4$. $\S 2$ is devoted to the proofs of Theorems $1-5$ while $\$ 3$ contains the proof of Theorem 6 .

Studying these problems for the last decade the author has benefitted from correspondence and conversations with J. Ralston, D. Tartakoff and L. Sarason. Thanks! 


\section{The $\mathscr{L}^{2}$ theory.}

Proof of Proposition 1. Only the density requires comment. Cover $\bar{\Omega}$ by finitely many coordinate patches $C^{1}$ diffeomorphic to $\{|x|<1\}$ or to $\left\{|x|<1\right.$ and $\left.x_{1}>0\right\}$ by diffeomorphisms $\chi_{i}$. Choose a finite smooth partition of unity $\phi_{i}$ subordinate to this cover. Choose $j \in C_{0}^{\infty}\left(|x|<1\right.$ and $\left.x_{1}<0\right), \int j=1$ and let $j_{\varepsilon}(x)=\varepsilon^{-n} j\left(\varepsilon^{-1} x\right)$. Let

$$
u_{\varepsilon}=\sum_{i}\left(j_{\varepsilon} *\left(\phi_{i} u \circ \chi_{i}^{-1}\right)\right) \circ \chi_{i} .
$$

Then $u_{\varepsilon} \in C^{1}(\bar{\Omega})$ and $u_{\varepsilon} \rightarrow u$ in $\mathscr{L}^{2}(\Omega)$. The classical lemma of Friedrichs [4] implies that $(L u)_{\varepsilon}-L\left(u_{\varepsilon}\right) \rightarrow 0$ in $\mathscr{L}^{2}(\Omega)$, so $L\left(u_{\varepsilon}\right) \rightarrow L u$ in $H^{1}(\Omega)^{\prime}$. Thus $u_{\varepsilon} \rightarrow u$ in $\mathscr{K}_{L}$.

Proof of Theorem 1. Given $\psi \in H^{1 / 2}(\partial \Omega)$ choose $\Psi \in H^{1}(\Omega)$ such that $\left.\Psi\right|_{\partial \Omega}=$ $\psi,\|\Psi\|_{H^{1}}<c\|\psi\|_{H^{1 / 2}}$ with $c$ independent of $\psi$. Then for $u \in C^{1}(\bar{\Omega})$,

$$
\int_{\partial \Omega}\left\langle A_{n} u, \psi\right\rangle d \sigma=\int_{\Omega}\left\langle u, L^{*} \Psi\right\rangle+\langle L u, \Psi\rangle d x .
$$

Thus

$$
\begin{aligned}
\left|\int_{\partial \Omega}\left\langle A_{n} u, \psi\right\rangle d \sigma\right| & \leqslant\|u\|_{\mathscr{L}^{2}(\Omega)}\left\|L^{*} \Psi\right\|_{\mathscr{L}^{2}(\Omega)}+\|L u\|_{H^{1}(\Omega)^{\prime}}\|\Psi\|_{H^{1}(\Omega)} \\
& \leqslant c\|\psi\|_{H^{1 / 2}(\partial \Omega)}\|u\|_{\mathscr{C}_{l}} .
\end{aligned}
$$

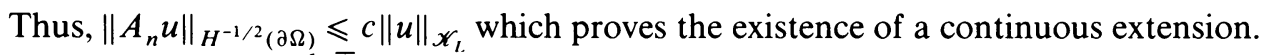
By Proposition $1, C^{1}(\bar{\Omega})$ is dense so the extension is unique.

Proof of Theorem 2. Given $\psi \in \operatorname{Lip}(\partial \Omega)$ choose $\Psi \in \operatorname{Lip}(\bar{\Omega})$ so that $\left.\Psi\right|_{\partial \Omega}=\psi$ and $\|\Psi\|_{\operatorname{Lip}(\bar{\Omega})} \leqslant \mathrm{c}\|\psi\|_{\operatorname{Lip}(\partial \Omega)}$ with $c$ independent of $\psi$. Then for $u, v \in C^{1}(\bar{\Omega})$, Green's identity yields

$$
\int_{\partial \Omega} \psi\left\langle A_{n} u, v\right\rangle d \sigma=\int_{\Omega}\langle L u, \Psi v\rangle+\left\langle u, L^{*}(\Psi v)\right\rangle d x .
$$

Since the commutator $\left[L^{*}, \Psi\right]$ is of order zero we see that with $c$ independent of $\psi$

$$
\left|\int \psi\left\langle A_{n} u, v\right\rangle d \sigma\right| \leqslant c\|\psi\|_{\operatorname{Lip}(\partial \Omega)}\|u\|_{\mathscr{H}_{L}}\|v\|_{\mathscr{H}_{L} .} .
$$

Thus $\left\|\left\langle A_{n} u, v\right\rangle\right\|_{\operatorname{Lip}(\partial \Omega)^{\prime}} \leqslant c\|u\|_{\mathscr{X}_{L}}\|v\|_{\mathscr{H}_{l} .}$. Since $C^{1}(\bar{\Omega})$ is dense, Theorem 2 follows.

Proof of Theorem 3. The proof of Theorem 2 shows that for $u \in C^{1}$

$$
\left\|\left.A_{1} u\right|_{x_{1}=s}\right\|_{H^{-1 / 2}\left(\mathbf{R}^{j-1}\right)} \leqslant c\|u\|_{\mathscr{X}_{L}}
$$

with $c$ independent of $u$ and s. For $u \in \mathscr{K}_{L}$, choose $u_{n} \in C^{1}$ with support in the coordinate patch and $u_{n} \rightarrow u$ in $\mathscr{K}_{L}$. By (15) $u_{n}$ is a Cauchy sequence in $C\left(\overline{\mathbf{R}}_{+}: H^{-1 / 2}\left(\mathbf{R}^{\nu-1}\right)\right)$, so there is $\tilde{u}$ such that $u_{n} \rightarrow \tilde{u}$ in $C\left(\overline{\mathbf{R}}_{+}: H^{-1 / 2}\left(\mathbf{R}^{\nu-1}\right)\right)$. Thus $u_{n} \rightarrow \tilde{u}$ in $\mathscr{D}^{\prime}\left(\mathbf{R}_{+}^{\nu}\right)$ so $u=\tilde{u}$. Thus $u \in C\left(\mathbf{R}_{+}: H^{-1 / 2}\left(\mathbf{R}^{\nu-1}\right)\right)$ and (15) holds for $u$.

Similarly, for $u, v \in C^{1}$, the proof of Theorem 2 yields

$$
\left\|\left.\langle A, u, v\rangle\right|_{x_{1}=s}\right\|_{\operatorname{Lip}\left(\mathbf{R}^{\nu-1}\right)^{\prime}} \leqslant c\|u\|_{\mathscr{F}_{l}}\|v\|_{\mathscr{X}_{L} \text {. }}
$$

with $c$ independent of $u, v$ and $s$. Using this in the same fashion as (15), the second part of Theorem 3 follows. 
Proof OF THEOREM 4. With the aid of a partition of unity write $u=\sum \phi_{i} u \equiv \sum u^{i}$. Then

$$
L u^{i}=\phi_{i} f+\sum c_{i j}(x) u^{j} \equiv f_{i} .
$$

If we can find $u_{\varepsilon}^{i}$ in $C^{1}(\bar{\Omega})$ supported near supp $\phi_{i}, u_{\varepsilon}^{i} \in N$ at $\partial \Omega$, and $u_{\varepsilon}^{i} \rightarrow u^{i}$ in $\mathscr{K}_{L}$ (resp. $\mathscr{H}_{L}$ ), then letting $u_{\varepsilon}=\sum u_{\varepsilon}^{i}$ gives the desired approximation for $u$. Thus, it suffices to consider $u$ supported in a small coordinate patch. The interesting patches are at $\partial \Omega$. Performing a change of independent variable we are reduced to the case $\Omega=\mathbf{R}_{+}^{n}, \operatorname{supp} u \subset\left\{|x|<1\right.$ and $\left.x_{1} \geqslant 0\right\}$.

Under a change of dependent variable, $\tilde{u}=M(x)^{-1} u$, the differential equation is transformed to $\tilde{L} \tilde{u}=M^{*} f$ where

$$
\tilde{L} v=\sum M^{*} A_{j} \partial_{j}(M w)+M * B M w .
$$

By hypothesis, $A_{1}\left(0, x^{\prime}\right)$ has rank independent of $x^{\prime}$ so by a Lipshitzean change, $M\left(x^{\prime}\right)$, we can transform $A_{1}\left(0, x^{\prime}\right)$ to

$$
\left[\begin{array}{ccc}
0 & 0 & 0 \\
0 & I & 0 \\
0 & 0 & -I
\end{array}\right] .
$$

Multiplying on the left by

$$
\left[\begin{array}{ccc}
I & 0 & 0 \\
0 & I & 0 \\
0 & 0 & -I
\end{array}\right]
$$

transforms to an equivalent, but nonsymmetric, system with

$$
A_{1}\left(0, x^{\prime}\right)=\left[\begin{array}{lll}
0 & 0 & 0 \\
0 & I & 0 \\
0 & 0 & I
\end{array}\right] .
$$

Since $N \supset \operatorname{ker} A_{1}$ we may choose a Lipshitz continuous unitary $U\left(x^{\prime}\right)$ such that $U$ leaves $\operatorname{ker} A_{1}$ invariant and $U^{*}\left(x^{\prime}\right)\left(N\left(x^{\prime}\right)\right)$ is equal to

$$
\left\{u \in \mathbf{C}^{k} \mid u_{l+1}=\cdots=u_{k}=0\right\} \equiv N_{0} .
$$

The change of dependent variable $\tilde{\tilde{u}}=U^{*} \tilde{u}$ transforms to an equivalent system with $A_{1}$ unchanged since $U^{*} A_{1} U=A_{1}$ and with the boundary space $N$ replaced by $N_{0}$. These changes have simultaneously transformed $A_{1}\left(0, x^{\prime}\right)$ to the form (16) and $N$ to $N_{0}$ in (17), both independent of $x^{\prime}$. We now drop the tildes and work with the transformed boundary value problem.

Suppose $u \in \mathscr{K}_{L}$ and $u \in N_{0}$ on $x_{1}=0$. The approximation $u_{\varepsilon}$ is made in three steps. First we construct $u_{\varepsilon} \in H_{\tan }^{1} \cap \mathscr{K}_{L}, u_{\varepsilon} \in N_{0}, u_{\varepsilon} \rightarrow u$ in $\mathscr{K}_{L}$. The construction uses a variant of Friedrichs's mollifiers. Choose $j \in C_{0}^{\infty}\left(\left\{|x|<1\right.\right.$ and $\left.\left.x_{1}>0\right\}\right), j \geqslant 0$ and $\int j=1$. Let

$$
u_{\varepsilon} \equiv J_{\varepsilon} u \equiv \int u\left(x_{1} e^{\varepsilon y_{1}}, x^{\prime}+\varepsilon y^{\prime}\right) j(y) d y .
$$

The novelty here is that instead of $x_{1}+\varepsilon y_{1}$ we have $x_{1} e^{\varepsilon y_{1}}$ which is the point $\varepsilon$ units of time along the integral curve of $x_{1} \partial / \partial x_{1}$ with initial point $x_{1}$. The gain is that $\left.u\right|_{x_{1}=0}$ is determined by $\left.u\right|_{x_{1}=0}$ and the loss is that the mollifier is not completely smoothing; one gains $\left(x_{1} \partial_{1}, \partial^{\prime}\right)$ derivatives but not $\partial_{1}$. A related idea, convoluting 
only in the $x^{\prime}$ variables, was used by Lax and Phillips [9]. For that method it appears necessary to suppose that $\operatorname{dim} \operatorname{ker} A_{1}$ is constant on a neigborhood of $x_{1}=0$.

Lemma. (1) With $X=H^{1}\left(\mathbf{R}_{+}^{\nu}\right)$ or $X=H_{\text {tan }}^{s}\left(\mathbf{R}_{+}^{\nu}\right)$ and $\phi \in X$ with compact support, $\left\{J_{\varepsilon} \phi\right\}_{0<\varepsilon<1}$ is a bounded subset of $X$. As $\varepsilon \rightarrow 0, J_{\varepsilon} \phi$ converges to $\phi$ in $X$.

(2) If $Z=\left(x_{1} \partial_{1}, \partial_{2}, \ldots, \partial_{\nu}\right)$ and $\phi$ is as above, then $Z^{\alpha} J_{\varepsilon} \phi \in X$ for all $\alpha \in \mathbf{Z}^{\nu}$.

(3) Suppose $s \geqslant 0$ is an integer, $\phi \in H_{\mathrm{tan}}^{s}\left(\mathbf{R}_{+}^{\nu}\right)$ with compact support and $A \in$ $C^{s, 1}\left(\left(\overline{\mathbf{R}_{+}^{\nu}}\right): \operatorname{Hom}\left(\mathbf{C}^{k}\right)\right)$. Then if $|\alpha|=1$ the family $\left[A Z^{\alpha}, J_{\varepsilon}\right] \phi, 0<\varepsilon<1$, is bounded in $H_{\mathrm{tan}}^{s}$ and as $\varepsilon \rightarrow 0,\left[A Z^{\alpha}, J_{\varepsilon}\right] \phi \rightarrow 0$ in $H_{\mathrm{tan}}^{s}$.

Proof of Lemma. (1) For $\phi \in X$ with compact support we have

$$
\phi\left(x_{1} e^{\varepsilon y_{1}}, x^{\prime}+\varepsilon y^{\prime}\right) \in X \text { for }|y| \leqslant 1,0 \leqslant \varepsilon \leqslant 1 .
$$

In fact they lie in a bounded subset of $X$. As $J_{\varepsilon} \phi$ is a convex combination it is bounded in $X$ uniformly in $\varepsilon \leqslant 1$, with bound depending only on $\|\phi\|_{X}$ and supp $\phi$. Approximating $\phi$ in $X$ by elements of $C_{(0)}^{1}\left(\overline{\mathbf{R}^{\nu}}\right)$ with uniformly bounded supports, part (1) follows since $J_{\varepsilon} \psi \rightarrow \psi$ in $X$ for $\psi \in C_{(0)}^{\infty}\left(\overline{\mathbf{R}_{+}^{\nu}}\right)$.

(2) Consider first $x_{1} \partial_{1}$. For $\phi \in C_{(0)}^{\infty}\left(\overline{\mathbf{R}_{+}^{\nu}}\right), J_{\varepsilon} \phi \in C_{(0)}^{\infty}\left(\overline{\mathbf{R}_{+}^{\nu}}\right)$ and differentiating under the integral sign

$$
\begin{aligned}
x_{1} \partial_{1} J_{\varepsilon} \phi & =\int x_{1} e^{\varepsilon y_{1}} \frac{\partial \phi}{\partial x_{1}}\left(x_{1} e^{\varepsilon y_{1}}, x^{\prime}+\varepsilon y^{\prime}\right) j(y) d y \\
& =\int j(y) \frac{1}{\varepsilon} \frac{\partial}{\partial y_{1}}\left(\phi\left(x_{1} e^{\varepsilon y_{1}}, x^{\prime}+\varepsilon y^{\prime}\right)\right) d y .
\end{aligned}
$$

Integrating by parts using the fact that $j=0$ when $y_{1}=0$ yields

$$
=-\frac{1}{\varepsilon} \int \phi\left(x_{1} e^{e y_{1}}, x^{\prime}+\varepsilon y^{\prime}\right) \frac{\partial j}{\partial y_{1}}(y) d y .
$$

More generally we have

$$
Z^{\alpha} J \varepsilon \phi=\left(\frac{-1}{\varepsilon}\right)^{|\alpha|} \int \phi\left(x_{1} e^{\varepsilon y_{1}}, x^{\prime}+\varepsilon y^{\prime}\right)\left(\partial_{y}^{\alpha} j\right)(y) d y .
$$

Thus for $\varepsilon$ and $\alpha$ fixed and $K \subset \overline{\mathbf{R}_{+}^{\nu}}$ compact there is $C=C(\varepsilon, \alpha, K)$ so that $\left\|Z^{\alpha} J_{\varepsilon} \phi\right\|_{X} \leqslant c\|\phi\|_{X}$. Approximating $\phi \in X$ with compact support by a sequence $\phi_{k} \in C_{(0)}^{\infty}$, (2) follows.

(3) We treat $\alpha=(1,0, \ldots, 0)$ and $s=0$. The other cases are similar. For $\phi \in$ $C_{(0)}^{\infty}\left(\overline{\mathbf{R}_{+}^{\nu}}\right)$ and $\Gamma \equiv Z^{\alpha}$ we have

$$
\left[A \Gamma, J_{\varepsilon}\right] \phi=\int\left(A(x)-A\left(x_{1} e^{\varepsilon y_{1}}, x^{\prime}+\varepsilon y^{\prime}\right)\right) j(y) \frac{1}{\varepsilon} \frac{\partial}{\partial y_{1}}\left(\phi\left(x_{1} e^{\varepsilon y_{1}}, x^{\prime}+\varepsilon y^{\prime}\right)\right) d y .
$$

Now integrate by parts. When the $y$ derivative falls on the $A$ term we find

$$
\int x_{1} e^{\varepsilon \nu_{1}} \frac{\partial A}{\partial x_{1}}\left(x_{1} e^{\varepsilon \nu_{1}}, x^{\prime}+\varepsilon y^{\prime}\right) j(y) \phi\left(x_{1} e^{\varepsilon y_{1}}, x^{\prime}+\varepsilon y^{\prime}\right) d y
$$


whose $\mathscr{L}^{2}\left(\mathbf{R}_{+}^{\nu}\right)$ norm is bounded independent of $\varepsilon \in(0,1]$ since $x_{1} \partial A / \partial x_{1}$ is bounded. When the $y$ derivative falls on $j$ we find

$$
-\int \frac{A(x)-A\left(x_{1} e^{\varepsilon y_{1}}, x^{\prime}+\varepsilon y^{\prime}\right)}{\varepsilon} \frac{\partial j}{\partial y_{1}}(y) \phi\left(x_{1} e^{\varepsilon y_{1}}, x^{\prime}+\varepsilon y^{\prime}\right) d y
$$

whose $\mathscr{L}^{2}\left(\mathbf{R}_{+}^{n}\right)$ norm is bounded independent of $\varepsilon \in(0,1]$ because the difference quotient in the integrand is bounded since $A \in \operatorname{Lip}$. Thus $\left[A \Gamma, J_{\varepsilon}\right]$ maps $\mathscr{L}^{2}\left(\mathbf{R}_{+}^{\nu}\right)$ to itself with norm independent of $\varepsilon \in(0,1]$. Since $\mathscr{L}^{2}-\lim \left[A \Gamma, J_{\varepsilon}\right] \phi=0$ for $\phi \in$ $C_{(0)}^{\infty}\left(\overline{\mathbf{R}_{+}^{\nu}}\right)$, a dense subset of $\mathscr{L}^{2}$, part (3) follows.

Applying part (2) of the lemma with $X=\mathscr{L}^{2}\left(\mathbf{R}_{+}^{\nu}\right)$ we see that $u_{\varepsilon} \equiv J_{\varepsilon} u \in H_{\tan }^{1}\left(\mathbf{R}_{+}^{\nu}\right)$. Part (1) with $X=H^{1}\left(\mathbf{R}_{+}^{\nu}\right)^{\prime}$ shows $J_{\varepsilon}(L u) \rightarrow L u$ in $H^{1}\left(\mathbf{R}_{+}^{\nu}\right)^{\prime}$. Finally, part (3) shows that $J_{\varepsilon}(L u)-L\left(u_{\varepsilon}\right) \rightarrow 0$ in $\mathscr{L}^{2}\left(\mathbf{R}_{+}^{\nu}\right)$. Putting this together we see that for $u \in \mathscr{K}_{L}$, $u_{\varepsilon} \rightarrow u$ in $\mathscr{K}_{L}$.

Since $N_{0}$ is independent of $x^{\prime}$, it is clear on a formal level that $u_{\varepsilon} \in N_{0}$ at $x_{1}=0$. To prove this, first observe that for $\phi \in C_{(0)}^{\infty}\left(\overline{\mathbf{R}_{+}}\right)$,

$$
\left.J_{\varepsilon} \phi\right|_{x_{1}=0}=\gamma_{\varepsilon} *\left(\left.\phi\right|_{x_{1}=0}\right), \quad \gamma_{\varepsilon}\left(x^{\prime}\right) \equiv \varepsilon^{-(\nu-1)} \gamma\left(x^{\prime} / \varepsilon\right), \quad \gamma\left(x^{\prime}\right) \equiv \int j\left(x_{1}, x^{\prime}\right) d x_{1},
$$

the convolution in $x^{\prime}$ variables only. Define $M_{0}: \mathbf{C}^{k} \rightarrow \mathbf{C}^{k} / N_{0}$ by the commutative diagram:

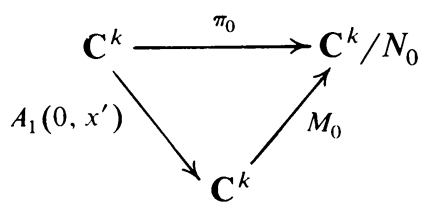

Since $M_{0}$ and $A_{1}$ are independent of $x^{\prime}$ we have

$$
\left.M_{0} A_{1} J_{\varepsilon} \phi\right|_{x_{1}=0}=\gamma_{\varepsilon} *\left(\left.M_{0} A_{1} \phi\right|_{x_{1}=0}\right),
$$

the convolution in $x^{\prime}$ variables. Using Theorem 1 we see that this identity extends by continuity to $\phi \in \mathscr{K}_{L}$, the equality expressed in $H^{-1 / 2}\left(\mathbf{R}^{\nu-1}\right)$. For $u \in \mathscr{K}_{L}$ with $u \in N$ at $\partial \Omega,\left.M_{0} A_{1} u\right|_{x_{1}=0}=0$ so we find that $M_{0} A_{1} u_{\varepsilon}=0$, that is $u_{\varepsilon} \in N_{0}$ at $x_{1}=0$.

Thus, replacing $u$ by $u_{\varepsilon}$ we may suppose without loss of generality that $u \in$ $H_{\text {tan }}^{1}\left(\mathbf{R}_{+}^{\nu}\right)$.

The differential equation and $u \in H_{\tan }^{1}$ imply that $\partial_{1}\left(A_{1} u\right) \in \mathscr{L}^{2}\left(\mathbf{R}_{+}^{\nu}\right)$, so $A_{1} u \in$ $H^{1}\left(\mathbf{R}_{+}^{\nu}\right)$. Let $u^{I I}=\left(0,0, \ldots, 0, u_{l+1}, \ldots, u_{k}\right)$ be the projection of $u$ orthogonal to $N_{0}$. Since $N_{0} \supset \operatorname{ker} A_{1}$ we see that $u^{I I} \in H^{1}\left(\mathbf{R}_{+}^{\nu}\right)$. The boundary condition, though expressed weakly, implies $u^{I I} \in \stackrel{H}{ }^{1}\left(\mathbf{R}_{+}^{\nu}\right)$. Extend $u^{I I}$ to be zero for $x_{1}<0$, so $u^{I I} \in H^{1}\left(\mathbf{R}^{\nu}\right)$. Let $u^{I}=u-u^{I I}$ and for $\eta \in(0,1]$

$$
u_{\eta}(x)=u^{I}(x)+u^{I I}\left(x_{1}-\eta, x^{\prime}\right) .
$$

Then as $\eta \rightarrow 0, u_{\eta}-u \rightarrow 0$ in $\left.H^{1}(\mathbf{R})_{+}^{\nu}\right)$, so $L u_{\eta} \rightarrow L u$ in $\mathscr{L}^{2}\left(\mathbf{R}_{+}^{\nu}\right)$. Thus $u_{\eta} \rightarrow u$ in $\mathscr{K}_{L}$. Replacing $u$ by $u_{\eta}$ we may suppose without loss of generality that $u^{I I}=0$ for $0<x_{1}<\eta$. 
With $j$ as before and $j_{\varepsilon}(x) \equiv \varepsilon^{-v} j\left(-x_{1} / \varepsilon, x / \varepsilon\right)$ and $\varepsilon<\eta$ let $u_{\varepsilon}=j_{\varepsilon} * u \in C_{(0)}^{\infty}\left(\overline{\mathbf{R}_{+}^{v}}\right)$, $\left(u_{\varepsilon}\right)^{I I}=0$ for $x_{1}<\eta-\varepsilon$. Thus $u_{\varepsilon} \in N_{0}$ at the boundary. That $u_{\varepsilon} \rightarrow u$ in $\mathscr{L}^{2}\left(\mathbf{R}_{+}^{\nu}\right)$ and $j_{\varepsilon} * L u \rightarrow L u$ in $H^{1}\left(\mathbf{R}_{+}^{\nu}\right)^{\prime}$ are routine. Friedrichs' classical lemma asserts that $j_{\varepsilon} * L u-L u_{\varepsilon} \rightarrow 0$ in $\mathscr{L}^{2}\left(\mathbf{R}_{+}^{\nu}\right)$. In total, $u_{\varepsilon} \rightarrow u$ in $\mathscr{K}_{L}$ and the proof of Theorem 4 for $\mathscr{K}_{L}$ is complete. For $\mathscr{H}_{L}$ one merely repeats the proof replacing $\mathscr{K}$ by $\mathscr{H}$ and $H^{1}\left(\mathbf{R}_{+}^{\nu}\right)^{\prime}$ by $\mathscr{L}^{2}\left(\mathbf{R}_{+}^{\nu}\right)$.

Proof OF Proposition 3. If $u \in \mathscr{K}_{L}$ with $u \in N$ at $\partial \Omega$ we may choose $u_{\varepsilon} \in \mathscr{K}_{L} \cap$ $C^{1}(\bar{\Omega}), u_{\varepsilon} \in N$ at $\partial \Omega$ and $u_{\varepsilon} \rightarrow u$ in $\mathscr{K}_{L}$. Green's identity for $u_{\varepsilon}$ yields

$$
\int_{\Omega}\left\langle u_{\varepsilon}, L^{*} v\right\rangle d x=\left(L u_{\varepsilon}\right)(v)+\int_{\partial \Omega}\left\langle A_{n} u_{\varepsilon}, v\right\rangle d \sigma .
$$

For $v \in \operatorname{Lip}(\partial \Omega)$ with $v \in N^{*}$ the boundary term vanishes. Passing to the limit $\varepsilon \rightarrow 0$ yields (8).

Conversely, suppose (8) holds for $u \in \mathscr{K}_{L}$. Then for any $\psi \in \operatorname{Lip}(\partial \Omega)$ with $\psi \in N^{*}$ we may choose $v \in \operatorname{Lip}(\bar{\Omega}),\left.v\right|_{\partial \Omega}=\psi$. Then identity (8) implies that $\left(\left.A_{n} u\right|_{a \Omega}\right)(\psi)=0$. Thus if $\pi_{N^{*}(x)}$ is the orthogonal projection in $\mathbf{C}^{k}$ onto $N^{*}$ we see that $\left.A_{n} u\right|_{\partial \Omega}$ annihilates $\pi_{N^{*}} v$ for any $v \in \operatorname{Lip}(\partial \Omega)$. Since $\pi_{N^{*}}$ is selfadjoint, this is equivalent to $\pi_{N^{*}}\left(\left.A_{n} u\right|_{\partial \Omega}\right)=0$ in $H^{-1 / 2}(\partial \Omega)$. Here we have used the fact that $\pi_{N^{*}}$ multiplies $H^{s}(\partial \Omega)$ to itself for all $|s| \leqslant 1$. Since $N \supset \operatorname{ker} A_{n}$, we have ker $\pi_{N^{*}} A_{n}=N$ for all $x \in \partial \Omega$. An argument like that in Proposition 2 then shows that for $u \in \mathscr{K}_{L}$, $u \in N$ at $\partial \Omega \Leftrightarrow \pi_{N^{*}}\left(\left.A_{n} u\right|_{\partial \Omega}\right)=0$ in $H^{-1 / 2}(\partial \Omega)$. We conclude that if (8) holds then $u \in N$ at $\partial \Omega$ and Proposition 3 is proved.

Proof of Theorem 5. If $u \in \mathscr{H}_{L}$ and $u \in N$ at $\partial \Omega$ we may choose $u_{\varepsilon} \in C^{1}(\bar{\Omega})$ with $u_{\varepsilon} \in N$ at $\partial \Omega$ and $u_{\varepsilon} \rightarrow u$ in $\mathscr{H}_{L}$. Then $\left.\left\langle A_{n} u_{\varepsilon}, u_{\varepsilon}\right\rangle\right|_{\partial \Omega} \geqslant 0$ and $\left\langle A_{n} u_{\varepsilon}, u_{\varepsilon}\right\rangle \rightarrow$ $\left\langle A_{n} u, u\right\rangle$ in $\operatorname{Lip}(\partial \Omega)^{\prime}$. This implies that $\left.\left\langle A_{n} u, u\right\rangle\right|_{\partial \Omega}$ is a positive distribution. Green's identity (5) with $v=u$ yields estimate (13), and, in particular, uniqueness.

To prove existence let $\mathscr{B}$ be the set of $v \in \operatorname{Lip}(\bar{\Omega})$ with $v \in N^{*}$ at $\partial \Omega$. Since $N^{*}$ is maximal positive we have $a\|v\|_{\mathscr{L}^{2}(\Omega)} \leqslant\left\|L^{*} v\right\|_{\mathscr{L}^{2}(\Omega)}$ for all $v \in \mathscr{B}$, in particular $L^{*}$ is a bijection from $\mathscr{B}$ to $\mathscr{R} \equiv L^{*}(\mathscr{B})$. Define $l: \mathscr{R} \rightarrow \mathrm{C}$ by

$$
L^{*} v \mapsto \int_{\Omega} v \bar{f} d x
$$

The estimate for $L^{*}$ yields $a|l(v)| \leqslant\|f\|_{\mathscr{L}^{2}(\Omega)}$. Riesz's theorem implies that there is a $u \in \mathscr{L}^{2}(\Omega)$ so that $l(w)=(w, u)_{\mathscr{L}^{2}(\Omega)}$ for all $w \in \mathscr{R}$. This is exactly identity (8) of Proposition 3. Considering $w=L^{*} v, v \in C_{0}^{\infty}(\Omega)$ we find $L u=f$. Proposition 3 shows $u \in N$ at $\partial \Omega$ so $u$ is the desired solution.

\section{Tangential regularity.}

Proof of TheOREM 6. We begin with the derivation of the a priori estimate (14). Let $f=(L+\lambda) u$. Cover $\bar{\Omega}$ by a finite family of open sets $\mathscr{U}_{i}$ so that either $\mathscr{U}_{i} \subset \subset \Omega$ or $\mathscr{U}_{i} \cap \bar{\Omega}$ is $C^{s, 1}$ diffeomorphic to $\left\{x_{1} \geqslant 0\right\} \cap\{|x|<1\}$ with $\partial \Omega$ mapping to $\left\{x_{1}=0\right\}$. Choose a finite partition of unity $\left\{\phi_{i}\right\}$ subordinate to this cover and let $u=\sum \phi_{i} u \equiv \sum u_{i}$. Changing coordinates in the boundary patches yields functions $u_{i} \circ \chi_{i}$ defined on $\left\{x_{1} \geqslant 0\right\} \cap\{|x|<1\}$ which we continue to call $u_{i}$. Each function $u_{i}$ satisfies an equation of the form

$$
\left(L_{i}+\lambda\right) u_{i}=\phi_{i} f \circ \chi_{i}+\sum_{j} c_{i, j}(x) u_{j}
$$


where $L_{i}$ is the operator expressed in the new coordinates and the matrices $c_{i j}$ are of class $C^{s-1.1}$. Taking the $\mathscr{L}^{2}$ scalar product with $u_{i}$ yields

$$
\left|\left(u_{i},\left(L_{i}+\lambda\right) u_{i}\right)\right| \leqslant c\|u\|_{\mathscr{L}^{2}}\left(\|(L+\lambda) u\|_{\mathscr{L}^{2}}+\|u\|_{\mathscr{L}^{2}}\right) .
$$

However, as $u_{i}$ satisfies the maximal positive boundary condition $u_{i} \in N \circ \chi_{i}$ on $x_{1}=0$, Green's identity implies that

$$
\operatorname{Re}\left(u_{i},\left(L_{i}+\lambda\right) u_{i}\right) \geqslant(\operatorname{Re} \lambda-c)\left\|u_{i}\right\|^{2} .
$$

Summing on $i$, we find constants $\omega_{0}, c_{0}$ so that

$$
\left(\operatorname{Re} \lambda-\omega_{0}\right)\left\|u{\| \mathscr{L}^{2}(\Omega)} \leqslant c_{0}\right\|(L+\lambda) u \|_{\mathscr{L}^{2}(\Omega)} .
$$

We want such an estimate for the tangential derivatives of $u$. The basic idea is to apply the $\mathscr{L}^{2}$ estimate to the tangential derivatives of $u$. There are two problems, first the tangential derivatives $\gamma u$ need not satisfy the boundary conditions and, second, $L \gamma u$ need not have $\mathscr{L}^{2}$ norm dominated by $\|u\|_{H_{\text {tan }}^{1}}$. To overcome these difficulties the problem is transformed to a convenient form.

Since $\operatorname{ker} A_{1}\left(0, x^{\prime}\right) \subset N\left(x^{\prime}\right)$ we may choose a unitary matrix valued function $U_{i}$ of class $C^{s, 1}$ so that

$$
\begin{aligned}
& U_{i}^{*}(N)=\left\{u \in \mathbf{C}^{k}: u_{l+1}=\cdots=u_{k}=0\right\} \equiv N_{0}, \\
& U_{i}^{*}\left(\operatorname{ker} A_{1}\right)=\left\{u \in \mathbf{C}^{k}: u_{\alpha+1}=\cdots=u_{k}=0\right\}
\end{aligned}
$$

with $\alpha \leqslant l$. Then $\tilde{u}_{i} \equiv U_{i}^{*} u_{i}$ satisfies the boundary condition $\tilde{u}_{i} \in N_{0}$ and $L_{i}+\lambda$ is transformed to $\tilde{L}_{i}+\lambda$, where

$$
\tilde{L}_{i}=\sum_{j} U_{i}^{*} A_{j} U_{i} \partial_{j}+\text { l.o.t. } \equiv \sum \tilde{A_{j}} \partial_{j}+\tilde{B}
$$

The symmetry of $\tilde{A}_{1}$ shows that

$$
A_{1}\left(0, x^{\prime}\right)=\left[\begin{array}{lcc}
0 & 0 & 0 \\
0 & {[\text { NONSING }}
\end{array}\right],
$$

where the $(k-\alpha) \times(k-\alpha)$ lower right-hand block is invertible.

What we have done is to transform the problem so that $N$ and $\operatorname{ker} A_{1}\left(0, x^{\prime}\right)$ are independent of $x^{\prime}$. It is worth noting that one cannot, in general, arrange that $N$ and $A_{1}\left(0, x^{\prime}\right)$ be constant. To see this consider $A_{1}$ constant and $N\left(x^{\prime}\right)$ which varies from dissipative to conservative with $x^{\prime}$.

For notational simplicity we drop the tildes. Next we examine the commutator of $L_{i}$ with tangential derivatives. In local coordinates, the tangential vector fields are generated by the $Z_{j}$, where

$$
\left(Z_{1}, Z_{2}, \ldots, Z_{\nu}\right) \equiv\left(x_{1} \partial_{1}, \partial_{2}, \ldots, \partial_{\nu}\right)
$$

The critical observation is that for each $Z_{j}$ and $L_{i}$ there are matrices $\Gamma_{\beta}, \Psi$ so that

$$
\left[L_{i}, Z_{j}\right]=\sum_{|\beta| \leqslant 1} \Gamma_{\beta} Z^{\beta}+\Psi L_{i}
$$


where $\Gamma_{\beta}$ and $\Psi$ are of class $C^{s-2.1}$ or $L^{\infty}$ depending on whether $s$ is greater than or equal to one. The crucial commutators are $\left[A_{1} \partial_{1}, Z_{j}\right]$. For $j>1,\left[A_{1} \partial_{1}, Z_{j}\right]=$ $\left(Z_{j} A_{1}\right) \partial_{1}$. Write $A_{1}\left(x_{1}, x^{\prime}\right)=A_{1}\left(0, x^{\prime}\right)+x_{1} G\left(x_{1}, x^{\prime}\right)$ with $G$ of class $C^{s-1,1}$. Then

$$
\left(Z_{j} A_{1}\right) \partial_{1}=\left(\partial_{j} A_{1}\left(0, x^{\prime}\right)\right) \partial_{1}+\left(\partial_{j} G\right) Z_{1}
$$

with $\partial_{j} G$ of class $C^{s-2.1}$ (resp. $L^{\infty}$ ) if $s>1$ (resp. $s=1$ ). Because of the special form (19) we have

$$
\partial_{j} A_{1}\left(0, x^{\prime}\right)=H\left(0, x^{\prime}\right) A_{1}\left(0, x^{\prime}\right)
$$

with $H$ of class $C^{s-1,1}$. Thus,

$$
\left(\partial_{j} A_{1}\left(0, x^{\prime}\right)\right) \partial_{1}=H A_{1} \partial_{1}-H G Z_{1} .
$$

Now $A_{1} \partial_{1}=L-\sum_{j=2}^{v} A_{j} Z_{j}-B$, so $\left[A_{1} \partial_{1}, Z_{j}\right]$ has the desired form for $j \geqslant 2$. For $j=1,\left[A_{1} \partial_{1}, Z_{1}\right]=A_{1} \partial_{1}-\left(\partial_{1} A_{1}\right) Z_{1}$. Replacing $A_{1} \partial_{1}$ as above completes the proof of (20).

We would like to apply the energy inequality to $Z_{l} u_{i}$. Dropping the subscripts we have

$$
(L+\lambda) Z u=Z(L+\lambda) u+[L, Z] u .
$$

For $f \in H_{\text {tan }}^{1}$ the middle term lies in $\mathscr{L}^{2}$ as does the last term by virtue of the commutator identity (20). Thus $Z_{l} u_{i} \in \mathscr{H}_{L}$. We need to know that $Z_{l} u_{i} \in N_{0}$ on $x_{1}=0$. Since $u_{i} \in N_{0}$ and $N_{0}$ is independent of $x^{\prime}$ this is obvious on the formal level. For proof consider $J_{\varepsilon} u_{i}$ as in the proof of Theorem 4. Using the lemma from that proof we see (subscripts dropped) that $J_{\varepsilon} u \rightarrow u$ in $H_{\tan }^{1}$ and $L J_{\varepsilon} u \rightarrow L u$ in $H_{\tan }^{1}$. It follows that $Z J_{\varepsilon} u \rightarrow Z u$ in $\mathscr{H}_{L}$ and therefore the equivalence classes $Z J_{\varepsilon} u \bmod N_{0}$ converge to $Z u$ mod $N_{0}$ in $H^{-1 / 2}\left(\mathbf{R}^{\nu-1}\right)$. Thus, it suffices to show that $Z J_{\varepsilon} u \in N_{0}$ at $x_{1}=0$. Since $N_{0}$ is independent of $x^{\prime}$ a simple calculation shows that for $u \in$ $C_{(0)}^{\infty}\left(\overline{\mathbf{R}_{+}}\right)$,

$$
Z J_{\varepsilon} u \bmod N=\gamma_{\varepsilon}\left(x^{\prime}\right) *\left(u \bmod N_{0}\right),
$$

where $\gamma_{\varepsilon}=-\varepsilon^{-\nu} \gamma\left(x^{\prime} / \varepsilon\right)$ and $\gamma\left(x^{\prime}\right)=\int_{-\infty}^{\infty}\left(Z_{l} j\right)\left(x_{1}, x^{\prime}\right) d x_{1}$. This identity extends by continuity to all $u \in \mathscr{K}_{L}$ with equality in $H^{-1 / 2}\left(\mathbf{R}^{\nu-1}\right)$. In particular, if $u \in N_{0}$, then $Z J_{\varepsilon} u \in N_{0}$.

The preliminaries complete, we apply the energy identity to $Z_{l} u_{i}$ to find

$$
\left(\operatorname{Re} \lambda-c_{0}\right)\left\|Z_{l} u_{i}\right\|_{\mathscr{L}^{2}\left(\mathbf{R}_{+}^{p}\right)}^{2} \leqslant c_{1}\left\|\left(L_{i}+\lambda\right) Z_{l} u_{i}\right\|_{\mathscr{L}^{2}\left(\mathbf{R}_{+}^{p}\right)}\left\|Z_{l} u_{i}\right\|_{\mathscr{L}^{2}\left(\mathbf{R}_{+}^{p}\right)} .
$$

Write $\left(L_{i}+\lambda\right) Z u=Z\left(L_{i}+\lambda\right) u+[L, Z] u$ and use (20) for the commutator. For the $L_{i} u$ term which arises write $L_{i} u=\left(L_{i}+\lambda\right) u-\lambda u$. This yields

$$
\left\|\left(L_{i}+\lambda\right) Z_{l} u_{i}\right\|_{\mathscr{L}^{2}\left(\mathbf{R}_{+}^{y}\right)} \leqslant c\left(\left\|\left(L_{i}+\lambda\right) u\right\|_{H_{\text {tan }}^{1}}+\|u\|_{H_{\text {an }}^{1}}+|\lambda|\|u\|_{\mathscr{L}^{2}}\right) .
$$

Plugging in and summing over $l$ and $i$ yields

$$
\left(\operatorname{Re} \lambda-c_{2}\right)\|u\|_{H_{\mathrm{tan}}^{1}}^{2} \leqslant c_{3}\|u\|_{H_{\mathrm{tan}}^{1}}\left(\|f\|_{H_{\mathrm{tan}}^{1}}+\|u\|_{H_{\mathrm{tan}}^{1}}+|\lambda|\|u\|_{\mathscr{L}^{2}}\right) .
$$

This is the desired estimate (14) for $s=1$. 
For higher $s$, one needs higher order commutators. Using (20) one proves by induction on $|\alpha|$ that for each $\alpha$ there are matrices $\Gamma_{\alpha, \beta}, \Psi_{\alpha, \beta}$ so that

$$
\begin{aligned}
& {\left[L_{i}, Z^{\alpha}\right]=\sum_{|\beta| \leqslant|\alpha|} \Gamma_{\alpha, \beta} Z^{\beta}+\sum_{|\beta|<|\alpha|} \Psi_{\alpha, \beta} Z^{\beta} L_{i},} \\
& \Gamma_{\alpha, \beta}, \Psi_{\alpha, \beta} \text { of class } \begin{cases}C^{s-|\alpha|-1,1} & \text { if } s>|\alpha|, \\
L^{\infty} & \text { if } s=|\alpha| .\end{cases}
\end{aligned}
$$

Then reasoning as above, one proves the a priori estimate (14) by induction on $s$.

We next turn to the proof of regularity. Assuming numbers $\lambda_{s}, c_{s}$ have been found so that (14) holds and that $f \in H_{\tan }^{s}$ and $\operatorname{Re} \lambda>\lambda_{s}$ we must show that the solution $u$ of $(L+\lambda) u=f, u \in N$ at $\partial \Omega$, lies in $H_{\tan }^{s}(\Omega)$. The proof proceeds in two steps. First we produce a number $\Lambda_{s}$ so that the conclusion holds provided $\operatorname{Re} \lambda>\Lambda_{s}$. The continuity method then yields the desired result for $\operatorname{Re} \lambda>\lambda_{s}$.

The first step proceeds by noncharacteristic regularization. Choose $n(x)$ as an extension of the unit outward normal to a $C^{s+1}$ vector field on $\bar{\Omega}$. Extend $N(x)$ to a $C^{s, 1}$ map defined on an open neighborhood of $\partial \Omega$, and choose $\phi \in C^{\infty}(\bar{\Omega})$ supported in the domain of definition of $N$ and equal to one at $\partial \Omega$. Let $\pi_{N(x)}$ be the orthogonal projection of $\mathbf{C}^{k}$ onto $N(x)$, and set

$$
L^{\varepsilon} \equiv L+\varepsilon \phi \pi_{N} \sum_{L=1}^{\nu} n_{i} \partial_{i} .
$$

For $L^{\varepsilon}$ with $\varepsilon$ small, $\partial \Omega$ is noncharacteristic and $N$ is a maximal positive boundary space. Actually, $N$ is strictly positive in the sense that (10) holds with strict inequality, $\left\langle A_{n}^{\varepsilon} v, v\right\rangle \geqslant \varepsilon|v|^{2}$ for $v \in N(x)$. A straightforward argument shows that there is an $\omega_{0}$ so that $L^{\varepsilon}+\lambda$ is positive for all $\operatorname{Re} \lambda>\omega_{0}$ and $0<\varepsilon<1$. Let $u^{\varepsilon}$ be the solution to $\left(L^{\varepsilon}+\lambda\right) u^{\varepsilon}=f, u^{\varepsilon} \in N$ at $\partial \Omega$. Then it is easy to see that as $\varepsilon \rightarrow 0, u^{\varepsilon}$ converges to $u$ in $\mathscr{L}^{2}(\Omega)$. In addition, if one retraces the derivation of the a priori $H_{\text {tan }}^{s}$ estimate one finds $\omega_{s}, c_{s}$ so that $\omega_{0} \leqslant \omega_{1} \leqslant \cdots$ and for all $u \in H_{\tan }^{s}$ with $L u^{\varepsilon} \in H_{\tan }^{s}$ and $u \in N$ at $\partial \Omega$,

$$
\left(\operatorname{Re} \lambda-\omega_{s}\right)\|u\|_{H_{\mathrm{tan}}^{1}} \leqslant c_{s}\left(\left\|\left(L^{\varepsilon}+\lambda\right) u\right\|_{H_{\mathrm{tan}}^{1}}+|\lambda|\|u\|_{H_{\mathrm{tan}}^{s-1}}\right) .
$$

From this and the fact that $\partial \Omega$ is noncharacteristic, we find the $H^{s}$ estimate

$$
\left(\operatorname{Re} \lambda-\omega_{s}\right)\|u\|_{H^{s}(\Omega)} \leqslant \frac{\tilde{c}_{s}}{\varepsilon}\left(\left\|\left(L^{\varepsilon}+\lambda\right) u\right\|_{H_{\mathrm{tan}}^{s}(\Omega)}+|\lambda|\|u\|_{H_{\text {tan }}^{s-1}}\right) .
$$

Lemma. There is an $\varepsilon_{s}>0$ so that if $\operatorname{Re} \lambda>\omega_{s}, 0<\varepsilon<\varepsilon_{s}$, and $f \in H^{s}(\Omega)$, the unique solution to $\left(L^{\varepsilon}+\lambda\right) u=f, u \in N$ at $\partial \Omega$, lies in $H^{s}(\Omega)$.

Remark. There are two reasons why we cannot merely apply the result of Tartakoff [23]. First, the coefficients of $L^{\varepsilon}$ and $\partial \Omega$ are not sufficiently regular and second, Tartakoff provides a $\Lambda_{s, \varepsilon}$ so that the regularity holds for $\operatorname{Re} \lambda>\Lambda_{s, \varepsilon}$. We need a constant $\Lambda$ independent of $\varepsilon$.

We postpone the proof of the lemma. Given the lemma and $\operatorname{Re} \lambda>\omega_{s}$, estimate (21) allows one to prove inductively that $\left\{u^{\varepsilon}\right\}$ is bounded in $H_{\tan }^{\sigma}$ for $\sigma=0,1,2, \ldots, s$. Since $u^{\varepsilon} \rightarrow u$ in $\mathscr{L}^{2}(\Omega)$, we conclude that $u \in H_{\tan }^{s}(\Omega)$. 
Finally, we must prove the same conclusion assuming $\operatorname{Re} \lambda>\lambda_{s}$, where $\lambda_{s}$ appearing in (14) may be smaller than $\omega_{s}$. Define a closed operator $L$ on $H_{\tan }^{s}(\Omega)$ by

$$
\mathscr{D}(L)=\left\{u \in H_{\mathrm{tan}}^{s}(\Omega) \mid L u \in H_{\mathrm{tan}}^{s}(\Omega) \text { and } u \in N \text { at } \partial \Omega\right\} .
$$

We have proved that $\left\{\operatorname{Re} \lambda>\omega_{s}\right\}$ lies in the resolvent set $\rho(L)$ of $L$. We must show that $\mathcal{O}=\left\{\operatorname{Re} \lambda>\lambda_{s}\right\}$ lies in $\rho(L)$. Now $\mathcal{O}$ is connected and $\mathcal{O} \cap \rho(L)$ is open. Thus, it suffices to show that $\mathcal{O} \cap \rho(L)$ is a closed subset of $\mathcal{O}$. Suppose $\mu_{k} \in \mathcal{O} \cap \rho(L)$ and $\mu_{k} \rightarrow \mu$ in $\mathcal{O}$. We must show that $\mu \in \rho(L)$. Inequality (14) implies that $Z(x) \geqslant\left(\operatorname{Re} \mu-\lambda_{s}\right) I$ for all $x \in \Omega$ where $Z(x)$ is the matrix appearing in (9). Thus, for any $f \in H_{\tan }^{s}(\Omega)$, there is a unique $u \in \mathscr{L}^{2}(\Omega)$ with $(L+\mu) u=f, u \in N$ at $\partial \Omega$. We need to show that $u \in H_{\text {tan }}^{s}(\Omega)$. Now

$$
u=\mathscr{L}^{2}-\lim u^{k}, \quad u^{k} \equiv\left(L+\mu_{k}\right)^{-1} f .
$$

By hypothesis $u^{k} \in H_{\tan }^{s}(\Omega)$. The a priori estimate (21) shows that $\left\{u^{k}\right\}$ is bounded in $H_{\text {tan }}^{s}$. It follows that $u \in H_{\text {tan }}^{s}(\Omega)$, the desired conclusion.

REMARK. Instead of noncharacteristic regularization one could prove tangential regularity directly using our mollifiers $J_{\varepsilon}$ in a proof imitating that of Tartakoff. Given Tartakoff's theorem the present path is shorter.

Proof of Lemma. Fix $\varepsilon$. We want to apply Tartakoff's theorem to $L^{\varepsilon}$, but the coefficients $\partial \Omega$ and $N$ are not smooth enough. Let $A_{j}, B$ be the coefficients of $L^{\varepsilon}$. Choose $A_{j}^{k}, B^{k} \in C^{\infty}(\bar{\Omega}), A_{j}^{k}$ symmetric so that as $k \rightarrow \infty, A_{j}^{k} \rightarrow A_{j}$, and $B_{j}^{k} \rightarrow B$ uniformly with $\left\{A_{j}^{k}\right\}$ and $\left\{B^{k}\right\}$ bounded in $C^{s, 1}(\bar{\Omega})$ and $C^{s-1,1}(\bar{\Omega})$, respectively. This yields operators $L^{\varepsilon, k}$ converging to $L^{\varepsilon}$.

Next choose $\Omega^{k} \subset \Omega$, increasing to $\Omega$ with $\partial \Omega^{k} \rightarrow \partial \Omega$ in the $C^{s+1}$ topology.

Finally choose boundary spaces $N^{k}$ defined in $\partial \Omega^{k}$, smooth and converging to $N$ in the $C^{s, 1}$ topology (which makes sense in a unique way). We may choose $N^{k}$ strictly positive and so that the strictly positive smooth problems $L^{\varepsilon, k}, \Omega^{k}, N^{k}$ satisfy $H^{s}\left(\Omega^{k}\right)$ estimates uniformly in $k$. That is, there are constants $\tilde{\omega}_{s}$ and $\tilde{C}_{s}$ so that for all $k$ and $u \in H^{s}\left(\mathbf{R}^{\nu}\right)$

$$
\left(\operatorname{Re} \lambda-\tilde{\omega}_{s}\right)\|u\|_{H^{s}\left(\Omega^{k}\right)} \leqslant \tilde{C}_{s}\left(\left\|\left(L^{\varepsilon, k}+\lambda\right) u\right\|_{H^{s}\left(\Omega^{k}\right)}+|\lambda|\|u\|_{H^{s-1}\left(\Omega^{k}\right)}\right) .
$$

For $\varepsilon, k$ fixed, the proof of Tartakoff's theorem provides a $\Lambda_{\varepsilon, k}$ so that if $\operatorname{Re} \lambda>\Lambda_{\varepsilon, k}$, then the solution $u^{\varepsilon, k}$ to $\left(L^{\varepsilon, k}+\lambda\right) u^{\varepsilon, k}=f, u^{\varepsilon, k} \in N^{k}$ at $\partial \Omega^{k}$, satisfies $u^{\varepsilon, k} \in H^{s}\left(\Omega^{k}\right)$. A continuity argument as the end of the proof of Theorem 6 implies that the same conclusion holds for $\operatorname{Re} \lambda>\tilde{\omega}_{s}$.

Now suppose that $\operatorname{Re} \lambda>\tilde{\omega}_{s}$. Then $\left\|u^{\varepsilon, k}\right\|_{H^{s}\left(\Omega^{k}\right)}$ is bounded independent of $k$. Choose extensions $u_{\text {ext }}^{\varepsilon, k}$ uniformly bounded in $H^{s}\left(\mathbf{R}^{\nu}\right)$. Let $u^{\varepsilon} \in H^{s}\left(\mathbf{R}^{\nu}\right)$ be a weak limit point. One shows easily that $\left.u^{\varepsilon}\right|_{\Omega}$ satifies the boundary value problem of the lemma. Again a continuity argument yields the same conclusion for all $\lambda$ with $\operatorname{Re} \lambda>\omega_{s}$. This completes the proof of the lemma and consequently of tangential regularity. 
4. Mixed initial boundary value problems. We are interested in solving

$$
\begin{cases}L u=F(t, x) & \text { in }(0, T) \times \Omega, \\ u(0, \cdot)=g & \text { in } \Omega, \\ U(t, x) \in N(t, x) & \text { for }(t, x) \in[0, T] \times \partial \Omega,\end{cases}
$$

where $N(t, x)$ is a Lipshitz continuous map from $[0, T] \times \partial \Omega$ to the subspaces of $\mathbf{C}^{k}$,

$$
\begin{aligned}
& L=\partial_{t}+\sum_{j=1}^{v} A_{j}(t, x) \partial_{j}+B(t, x), \\
& \qquad A_{j} \in \operatorname{Lip}([0, T] \times \bar{\Omega}), B \in L^{\infty}([0, T] \times \Omega),
\end{aligned}
$$

and $\partial \Omega$ is assumed to be of class $C^{1}$. We introduce the notations $I \equiv(0, T)$ $\mathcal{O} \equiv(0, T) \times \Omega$ and $\Gamma \equiv(0, T) \times \partial \Omega$.

Though $\partial \mathcal{O}$ has corners it is Lipshitzean so that $H^{\sigma}(\partial \mathcal{O})$ is well defined for all $|\sigma| \leqslant 1$. Hilbert spaces $\mathscr{K}_{L}, \mathscr{H}_{L}, \mathscr{K}_{L^{*}}, \mathscr{H}_{L^{*}}$ are defined as in $\S 1$ with $\mathcal{O}$ replacing $\Omega$. Again $C^{1}(\overline{\mathcal{O}})$ is dense in each. The next result is the analogue of Theorems 1 and 2. The proof is exactly as before.

THEOREM 7. The map

$$
\begin{gathered}
C^{1}(\overline{\mathcal{O}}) \ni u \mapsto \tau \in \mathscr{L}^{\infty}(\partial \mathcal{O}), \\
\tau= \begin{cases}u & \text { on }\{T\} \times \Omega, \\
-u & \text { on }\{0\} \times \Omega, \\
A_{n} u & \text { on } \Gamma\end{cases}
\end{gathered}
$$

extends uniquely to a continuous map from $\mathscr{K}_{L}$ to $H^{1 / 2}(\partial \mathcal{O})^{\prime}$. The map

$$
\begin{gathered}
C^{1}(\overline{\mathcal{O}}) \times C^{1}(\overline{\mathcal{O}}) \ni(u, v) \mapsto \rho \in \mathscr{L}^{\infty}(\partial \mathcal{O}), \\
\rho= \begin{cases}\langle u, v\rangle & \text { on }\{T\} \times \Omega, \\
-\langle u, v\rangle & \text { on }\{0\} \times \Omega, \\
\left\langle A_{n} u, v\right\rangle & \text { on } \Gamma\end{cases}
\end{gathered}
$$

extends uniquely to a continuous map from $\mathscr{H}_{L} \times \mathscr{H}_{L^{*}}$ to $\operatorname{Lip}(\partial \mathcal{O})^{\prime}$.

For $u \in \mathscr{K}_{L},\left.A_{n} u\right|_{\Gamma}$ is a distribution on $\Gamma$ which has an extension to an element of $H^{-1 / 2}(\partial \mathcal{O}) \equiv H^{1 / 2}(\partial \mathcal{O})^{\prime}$. It follows that $A_{n} u \in H^{1 / 2}(\Gamma)^{\prime} \equiv \stackrel{\circ}{H}^{-1 / 2}(\Gamma)$. If $N \supset \operatorname{ker} A_{n}$ we then find that $u \bmod N$ is a well defined element of $H^{1 / 2}\left(\Gamma: \mathbf{C}^{k} / N\right)^{\prime}$. When $u$ mod $N$ vanishes we say that $u \in N$ on $\Gamma$. In the same way the restrictions of $u$ to $\{t=0\} \times \Omega$ and $\{t=T\} \times \Omega$ are well defined elements of $H^{1 / 2}(\Omega)^{\prime}$. Next, we impose the hypothesis that $\Gamma$ is characteristic of constant multiplicity in the same sense that $\operatorname{dim} k e r A_{n}$ is constant on each component of $\Gamma$.

THEOREM 8. If $\partial \Gamma$ is characteristic of constant multiplicity, $u \in \mathscr{K}_{L}\left(\right.$ resp. $\left.\mathscr{H}_{L}\right)$ and $u \in N$ on $\Gamma$, then there is a sequence $u_{k} \in C^{1}(\overline{\mathcal{O}})$ such that $u_{k} \in N$ on $\Gamma$ and $u_{k} \rightarrow u$ in $\mathscr{K}_{L}\left(\right.$ resp. $\left.\mathscr{H}_{L}\right)$. In addition, if $u=0$ on $\{t=0\} \times \Omega$, then the $u_{k}$ may be chosen with $\left.u_{k}\right|_{t=0}=0$ on $\Omega$. 
OUTLINE OF PROOF. The proof is by mollification as in Theorem 4 but one must mollify in $x$ and $t$. By a partition of unity, one reduces to $u$ of small support. If $u$ vanishes near $\{t=0\} \times \bar{\Omega}$ and $\{t=T\} \times \bar{\Omega}$, one mollifies in $x$ as before and then in $t$. For $u$ supported near $\{t=0\}$ the mollification in time is performed with a kernel $j$ supported in $t>0$. Near $t=T$ one takes supp $j \subset\{t<0\}$. See [9, §4] for a similar calculation.

For the second part of the theorem, let $F \equiv L u$. Extend the coefficients of $L$ and the boundary space $N$ to $(-\infty, T] \times \bar{\Omega}$ by taking them independent of $t$ for $t<0$. Extend $u$ and $F$ to be equal to zero for $t<0$. Denote with a subscript $e$ the extended quantities. Since $u=0$ when $t=0$, we find $L_{e} u_{e}=F_{e}$.

Viewing $u_{e}$ as an element of $\mathscr{H}_{L_{e}}((-\infty, T) \times \Omega)$ we see that it can be approximated by elements in $C^{1}((-\infty, T] \times \bar{\Omega})$ obtained by mollification. The main point is that no special attention must be payed at $\{t=0\}$. One may mollify in $x$ and then in $t$ with any kernel. Choosing a kernel $j$ supported in $t>0$ for the contribution near $\{t=0\}$ yields approximations supported in $\{t>0\}$

We next suppose that $N$ is maximal positive, that is, (10) and (11) hold on $\bar{\Gamma}$. With

$$
Z \equiv \frac{B+B^{*}}{2}-\sum_{j=1}^{\nu} \partial_{j} A_{j} \in L^{\infty}(\mathcal{O})
$$

we have for $u \in C^{1}(\overline{\mathcal{O}}), F \equiv L u$,

$$
\frac{d}{d t}\|u(t)\|_{\mathscr{L}^{2}(\Omega)}^{2}+2(u, Z u)_{\Omega}=2 \operatorname{Re}(u, F)-2 \int_{\partial \Omega}\left\langle A_{n} u, u\right\rangle d \sigma .
$$

If $u \in N$ on $\Gamma$ the boundary integral is positive. With $c=\|Z\|_{\mathscr{L}^{\infty}(\mathcal{O})}$ and $\phi(t) \equiv$ $\|u(t)\|_{\mathscr{L}^{2}(\Omega)}$,

$$
\frac{d}{d t} \phi^{2}(t) \leqslant 2 \phi(t)\left(\|F(t)\|_{\mathscr{L}^{2}(\Omega)}+c \phi(t)\right) .
$$

It follows that

$$
\begin{gathered}
\sup _{0 \leqslant t \leqslant T} \phi(t) \leqslant c \| F{\| \mathscr{L}^{1}\left(I: \mathscr{L}^{2}(\Omega)\right)}+\phi(0), \\
\phi\left(t_{2}\right)-\phi\left(t_{1}\right) \leqslant \int_{t_{1}}^{t_{2}}\|F(\sigma)\|_{\mathscr{L}^{2}(\Omega)}+c \phi(\sigma) d \sigma
\end{gathered}
$$

with new constants $c$ independent of $u$. These estimates suggest the following theorem.

TheOREM 9 ( $\mathscr{L}^{2}$ WeLl - POSEDNeSS). For any $F \in \mathscr{L}^{1}\left(I: \mathscr{L}^{2}(\Omega)\right), g \in \mathscr{L}^{2}(\Omega)$, there is a unique $u \in \mathscr{L}^{2}(\mathcal{O})$ satisfying (22). In addition, $u \in C\left(I: \mathscr{L}^{2}(\Omega)\right)$ and with $\phi(t) \equiv\|u(t)\|_{\mathscr{L}^{2}(\Omega)}$, estimates (23) and (24) hold.

Proof of Uniqueness. The difference $\delta$ of two solutions lies in $\mathscr{H}_{L}$, satisfies the boundary condition on $\Gamma$, and vanishes on $\{t=0\}$. Use the second part of Theorem 8 to construct approximations $\delta_{k} \in C^{1}(\overline{\mathcal{O}})$ converging to $\delta$ in $\mathscr{H}_{L}$. Estimate (23) implies that $\delta_{k} \rightarrow 0$ in $\mathscr{L}^{2}(\mathcal{O})$, hence $\delta=0$.

Proof OF EXISTENCE. A simple approximation argument shows that it suffices to prove the existence of solutions $u$ when $g \in C_{0}^{\infty}(\Omega)$. For such $g$, subtracting a 
smooth function from $u$ reduces to the case $g=0$. If we can construct a solution to this problem with $u \in \mathscr{H}_{L}$, then the fact that $u \in C\left(I: \mathscr{L}^{2}(\Omega)\right)$ and satisfies (23), (24) follows by a simple approximation argument using the second part of Theorem 8. Thus, it suffices to construct $u \in \mathscr{H}_{L}$ with $L u=F,\left.u\right|_{t=0}=0$ and $u \in N$ on $\Gamma$.

Fix $F \in \mathscr{L}^{2}(\mathcal{O})$. The adjoint boundary space $N^{*} \equiv A_{n}(N)^{\perp}$ is Lipshitzean since $\Gamma$ is characteristic of constant multiplicity. Let

$$
\mathscr{B} \equiv\left\{v \in \operatorname{Lip}(\overline{\mathcal{O}}) \mid v \in N^{*} \text { on } \Gamma \text { and }\left.v\right|_{t=T}=0\right\} .
$$

Let $\mathscr{R} \equiv L^{*}(\mathscr{B})$. As $N^{*}$ is also maximal positive an inequality analogous to (23) shows that $L^{*}$ is one-to-one on $\mathscr{B}$ and that $\|w\|_{\mathscr{L}^{2}(\Omega)} \leqslant c\left\|L^{*} w\right\|_{\mathscr{L}^{2}(\mathcal{O})}$ with $c$ independent of $w \in \mathscr{B}$. Define $l: \mathscr{R} \rightarrow \mathbf{C}$ by

$$
\mathscr{R} \ni L^{*} w \mapsto \int_{0}\langle w, F\rangle \equiv l\left(L^{*} w\right)
$$

Then for any $r=L^{*} w \in \mathscr{R}$ we have

$$
|l(r)| \leqslant\|w\| \mathscr{L}^{2}(\mathcal{O})\|F\|_{\mathscr{L}^{2}(\mathcal{O})} \leqslant c\|r\|_{\mathscr{L}^{2}(\mathcal{O})}\|F\|_{\mathscr{L}^{2}(\mathcal{O})} .
$$

It follows that there is a $u \in \mathscr{L}^{2}(\mathcal{O})$ such that $l(r)=(r, u)_{\mathscr{L}^{2}(\mathcal{O})}$ for all $r \in \mathscr{R}$. Choosing $r=L^{*} \psi$ with $\psi \in C_{0}^{\infty}(\mathcal{O})$ we see that $L u=F$ in $\mathcal{O}$ so $u \in \mathscr{H}_{L}$. Let $\tau \in \operatorname{Lip}(\partial \theta)^{\prime}$ be the distribution which is formally equal to $u$ on $t=T,-u$ on $t=0$ and $\left\langle A_{n} u, u\right\rangle$ on $\Gamma$. The identity satisfied by $u$ shows that $\tau\left(\left.v\right|_{\partial \Theta}\right)=0$ for all $v \in \mathscr{B}$. Thus, $\tau(f)=0$ for all $f \in \operatorname{Lip}(\partial \mathcal{O})$ which vanish on $t=T$ and lie in $N^{*}$ on $\Gamma$. Choosing $f$ supported in $\{t=0\}$ we see that supp $\left.u\right|_{t=0} \subset \partial \Omega$. Similarly choosing $f$ supported in $\Gamma$ we find that $\operatorname{supp}(u \bmod N) \subset \partial \Gamma$. Now $\left.u\right|_{t=0}$ is an element of $H^{-1 / 2}(\Omega)$, and $u \bmod N$ is an element of $H^{-1 / 2}\left(\Gamma: \mathbf{C}^{k} / N\right)$. The only such distributions supported on the boundaries are the zero elements. This well-known fact is proved by localizing and then applying the following lemma.

Lemma. If $S \in \mathscr{E}^{\prime}\left(\mathbf{R}^{\nu}\right) \cap H^{-1 / 2}\left(\mathbf{R}^{\nu}\right)$ with $\operatorname{supp} \mathscr{S} \subset\left\{x_{1}=0\right\}$, then $\mathscr{S}=0$.

Proof. Let $m \geqslant 0$ be the order of $S$ and introduce the notation $x_{1}=\left(x_{1}, x^{\prime}\right)$, $\xi=\left(\xi_{1}, \xi^{\prime}\right)$. Then

$$
\left\langle S, e^{-i x \cdot \xi}\right\rangle=\left\langle S, e^{-x^{\prime} \cdot \xi^{\prime}} \sum_{j=0}^{m} \frac{\left(i x_{1} \xi_{1}\right)^{j}}{j !}\right\rangle .
$$

Thus $\hat{S}=\sum_{j=0}^{m} \xi_{1}^{j} f_{j}\left(\xi^{\prime}\right)$, where $f_{j} \in C^{\infty}\left(\mathbf{R}^{\nu-1}\right)$ is given by

$$
f_{j}\left(\xi^{\prime}\right)=\left\langle S, \frac{\left(i x_{1}\right)^{j}}{j !} e^{-i x^{\prime} \cdot \xi^{\prime}}\right\rangle .
$$

One easily shows that if $f_{m}\left(\overline{\xi^{\prime}}\right) \neq 0$, then for any $r>0$

$$
\int_{0}^{\infty} \int_{\left|\xi^{\prime}-\overline{\xi^{\prime}}\right|<r}|\hat{S}(\xi)|^{2}\langle\xi\rangle^{2 s} d \xi^{\prime} d \xi_{1}=\infty,
$$

Unless $2 s+2 m<-1$. By hypothesis the integral is finite for $s=-1 / 2$ so $f_{m}$ must vanish identically. A simple recursion then yields $f_{m-1}=f_{m-2}=\cdots=f_{0}=0$, whence $S=0$. 
We next study the regularity of solutions. For $s \in \mathbf{Z}_{+}$we seek solutions with derivatives up to order $s$. Toward this end we suppose that $A_{j}, N$, and $\partial \Omega$ are of Lipshitz class $C^{s, 1}$ and $B$ is of class $C^{s-1,1}$. One localizes as in $\S 2$ so that $N$ and $\operatorname{ker} A_{1}$ are independent of $x^{\prime}$, then applies the standard energy method to $\left(\partial_{t}, x_{1} \partial x_{1}, \partial x_{2}, \ldots, \partial x_{n}\right)^{\alpha} u$ with commutators controlled as in $\S 2$. Letting

$$
\phi_{s}(t) \equiv \sum_{j=0}^{s}\left\|\partial_{t}^{j} u(t)\right\|_{H_{\mathrm{tan}(}^{i^{\prime}(\Omega)}}
$$

one finds

$$
\begin{gathered}
\sup _{0 \leqslant t \leqslant T} \phi_{s}(t) \leqslant c\left(\sum_{j=0}^{s}\left\|\partial_{t}^{j} F\right\|_{\mathscr{L}^{1}\left(I: H_{\mathrm{tan}}^{\mathrm{i}-}(\Omega)\right)}+\phi_{s}(0)\right), \\
\phi_{s}\left(t_{2}\right)-\phi_{s}\left(t_{1}\right) \leqslant c \int_{t_{1}}^{t_{2}} \phi_{s}(t)+\sum_{j=0}^{s}\left\|\partial_{t}^{j} F(t)\right\|_{H_{\mathrm{tan}}^{\mathrm{s}-1}(\Omega)} d t .
\end{gathered}
$$

In addition to regularity of $F, g$ we must impose compatibility conditions at the corner $\{t=0\} \times \partial \Omega$. These conditions are computed in the usual fashion. For $(t, x) \in \bar{\Gamma}$, let $\pi(t, x)$ be the orthogonal projection of $\mathbf{C}^{k}$ onto $N(t, x)^{\perp}$. The compatibility condition of order $j$ comes from expressing $\partial_{t}^{j}(\pi u)$ at $\{t=0\} \times \partial \Omega$ in terms of $g$ and $F$ and requiring that the resulting expression vanishes. For example, for $j=0,1$ we find $t=0$

$$
\partial_{t}^{0}(\pi u)=\pi g, \quad \partial_{t}^{1}(\pi u)=\pi(F(0, \cdot)-G g)+\pi_{t} g
$$

where $L=\partial_{t}+G$. The compatibility conditions of order zero and one are

$$
\pi g=0 \quad \text { on } \partial \Omega, \quad \pi(F(0, \cdot)-G g)+\pi_{t} g=0 \quad \text { on } \partial \Omega .
$$

There is a subtle problem with the compatibility conditions. We illustrate this by considering the condition of order zero. If one seeks $u \in \bigcap_{j=0}^{1} C^{j}\left(\bar{I}: H_{\tan }^{1-j}(\Omega)\right)$ the a priori estimates suggest

$$
g \in H_{\tan }^{1}(\Omega), \quad F \in \bigcap_{j=0}^{1} \mathscr{L}^{1}\left(I: H_{\tan }^{1-j}(\Omega)\right), \quad \pi g=0 \quad \text { in } \partial \Omega
$$

as the natural conditions on the data. However, for data as above, the trace $\left.\pi g\right|_{\partial \Omega}$ is not defined. This is easily seen with the example on $\overline{\mathbf{R}}_{+}$given by the function $\left.\psi(x) \ln x, \psi \in C_{(0)}^{\infty} \overline{\mathbf{R}}_{+}\right), \psi(0) \neq 0$, which lies in $\bigcap_{s} H_{\text {tan }}^{s}\left(\overline{\mathbf{R}}_{+}\right)$and has no trace. On the other hand if $u \in \bigcap_{j=0}^{1} C^{j}\left(I: H_{\tan }^{1-j}(\Omega)\right)$ satisfies $L u=F$, where $F$ has the regularity suggested above, it follows that $\left.\pi u(0, \cdot)\right|_{\partial \Omega}$ is well-defined element of $H^{1 / 2}(\partial \Omega)$. Postponing the demonstration for a moment, we see that it is necessary to require more than $g \in H_{\tan }^{1}(\Omega)$. To prove that $\pi u(0, \cdot)$ has a trace, one localizes with a partition of unity and changes and independent variable, reducing to the case $[0, T] \times \mathbf{R}_{+}^{\nu}$. Then one finds $A_{1} u \in C\left(\bar{I}: H_{\text {tan }}^{1}\right)$ and, from the differential equation, $\partial_{1}\left(A_{1} u\right) \in C\left(\bar{I}: \mathscr{L}^{2}\right)$ since $F \in W^{1.1}\left(I: \mathscr{L}^{2}(\Omega)\right)$. Thus

$$
\left.A_{\nu} u\right|_{\partial \Omega} \in C\left(\bar{I}: H^{1 / 2}(\partial \Omega)\right),
$$

and it follows that $\left.\pi u(0, \cdot)\right|_{\partial \Omega} \in H^{1 / 2}(\partial \Omega)$. 
We are not able to describe the precise set of data $g, F$ leading to solutions in $\bigcap_{j=0}^{s} C^{j}\left(\bar{I}: H_{\tan }^{s-j}(\Omega)\right)$. However, we give an important subclass by assuming $H^{s}$ regularity in place of $H_{\tan }^{s}$ regularity near $t=0$.

Theorem 10 (TANGential Regularity). Suppose $s \geqslant 1$ is an integer, $A_{j}, N, \partial \Omega$ are of class $C^{s, 1}$ and $B$ is of class $C^{s-1,1}$. Suppose the data $g \in H^{s}$ and $\partial_{t}^{j} F \in$ $\mathscr{L}^{1}\left(I: H_{\tan }^{s-j}(\Omega)\right)$ for $0 \leqslant j \leqslant s$ and in addition there is a $0<T^{\prime} \leqslant T$ such that $\partial_{t}^{j} F \in \mathscr{L}^{1}\left(\left[0, T^{\prime}\right]: H^{s-j}(\Omega)\right), 0 \leqslant j \leqslant s$. If the data satisfy the compatibility conditions up to order $s-1$, then the solution $u$ to (22) lies in $\bigcap_{j=0}^{s} C^{j}\left(\bar{I}: H_{\tan }^{s-j}(\Omega)\right)$ and satisfies the estimates (25), (26).

Remark. With $g, F$ as in the theorem the compatibility conditions make sense. We check the cases $s=0,1$. For $s=0$, the condition is $\left.\pi_{N^{+}} g\right|_{\partial \Omega}=0$ and we have $g \in H^{1}(\Omega)$ so $\left.\pi_{N^{+}} g\right|_{\partial \Omega}$ is a well-defined element of $H^{1 / 2}(\partial \Omega)$. For $s=1$, we need the condition of order 1 which makes sense provided $\left.F(0, \cdot)\right|_{\partial \Omega}$ and $\left.G g\right|_{\partial \Omega}$ make sense. Here $g \in H^{2}(\Omega)$ so the second term is ok. For the first we observe that

$$
F \in W^{1,1}\left(I: H^{1}(\Omega)\right) \subset C\left(I: H^{1}(\Omega)\right)
$$

so the trace of $F$ on $\{t=0\} \times \partial \Omega$ lies in $H^{1 / 2}(\partial \Omega)$. The higher order conditions are similar.

Proof of TheOREM 10. As in the proof of Theorem 6 we make a noncharacteristic regularization, replacing $L$ by

$$
L^{\varepsilon}=L+\varepsilon \phi \pi_{N} \sum n_{j} \frac{\partial}{\partial x j} .
$$

For this operator $\partial \Omega$ is noncharacteristic and the boundary space is strictly positive. One then approximates $L^{\varepsilon}$ by operators $\tilde{L}^{\varepsilon}$, with smooth coefficients, $\Omega$ by smooth domains $\Omega^{\varepsilon}$, and $N$ by smooth strictly positive boundary spaces $N^{\varepsilon}$ so that estimates hold uniformly in $\varepsilon$. For the mixed problem we encounter a new difficulty. The data $F, g$ will not, in general, satisfy the compatibility condition for the regularized problem. In addition, $F$ is not smooth enough to apply directly the results in the literature. To solve the second dilemma choose $F^{\varepsilon} \in C_{(0)}^{\infty}(\overline{\mathcal{O}})$ such that

$$
\partial_{t}^{j} F^{\varepsilon} \rightarrow \partial_{t}^{j} F \quad \text { in } L^{1}\left(\left[0, T^{\prime}\right]: H^{s-j}(\Omega)\right) \cap L^{1}\left([0, T]: H_{\tan }^{s-j}(\Omega)\right)
$$

for $0 \leqslant j \leqslant s$. The final approximation $g^{\varepsilon} \in C_{(0)}^{\infty}(\Omega)$ must be done with care so as to ensure that the compatibility conditions are satisfied.

Lemma. One can choose $g^{\varepsilon} \in C_{(0)}^{\infty}(\bar{\Omega})$ so that $g^{\varepsilon} \rightarrow g$ in $H^{s}(\Omega)$ and the compatibility conditions up to order $s-1$ are satisfied by $\tilde{L}^{\varepsilon}, N^{\varepsilon}, \Omega^{\varepsilon}, F^{\varepsilon}, g^{\varepsilon}$.

Proof. The construction is local. We localize then introduce coordinates in $\mathbf{R}^{\nu}$ and $\mathrm{C}^{k}$ so that $\Omega^{\varepsilon}=\left\{x_{1}<\varepsilon\right\}, N^{\varepsilon}=\left\{u_{l+1}=\cdots=u_{k}=0\right\}$. Then since $N^{\varepsilon} \supset \operatorname{ker} A_{1}^{\varepsilon}$, the last $k-l$ rows of $A_{1}^{\varepsilon}$ form a matrix of rank $k-l$. The compatibility conditions for $g$ are

$$
\begin{aligned}
g & \equiv 0 \bmod N, \\
G g+F & \equiv 0 \bmod N, \quad \text { on } \partial \Omega . \\
G^{2} g+\left[G F+F_{t}+G_{t} u\right] & \equiv 0 \bmod N
\end{aligned}
$$


If one approximates $g$ by $g_{1}^{\varepsilon}$ in $H^{s}$ one finds that

$$
\begin{aligned}
g_{1}^{\varepsilon} & \equiv \phi_{0}^{\varepsilon} \bmod N^{\varepsilon} \\
G g_{1}^{\varepsilon}+F^{\varepsilon} & \equiv \phi_{1}^{\varepsilon} \bmod N^{\varepsilon} \quad \text { on } \partial \Omega
\end{aligned}
$$

with $\phi_{0}^{\varepsilon} \in C_{i}^{\infty}\left(\partial \Omega^{\varepsilon}\right), \phi_{i}^{\varepsilon}=o(1)$ in $H^{s-1 / 2-i}\left(\partial \Omega^{\varepsilon}\right)$ as $\varepsilon \rightarrow 0$. We will choose $g^{\varepsilon}=g_{1}^{\varepsilon}+$ $g_{2}^{\varepsilon}, g_{2}^{\varepsilon}$ chosen with its first $l$ components identically zero. The first compatibility condition for $g^{\varepsilon}$ requires $g_{2}^{\varepsilon} \equiv-\phi_{0}^{\varepsilon}$ mod $N^{\varepsilon}$ which determines the trace of $g_{2}^{\varepsilon}$ on $\partial \Omega^{\varepsilon}$. Given this trace the second compatibility condition determines the trace of $\partial g_{2}^{\varepsilon} / \partial x_{1}$ since the last rows of $A_{1}$ are of maximal rank. Continuing we see that to satisfy the compatibility conditions we must choose $g_{2}^{\varepsilon}$ so that

$$
\left(\frac{\partial}{\partial x_{1}}\right)^{j} g_{2}^{\varepsilon} \equiv \psi_{j}^{\varepsilon} \bmod N^{\varepsilon} \quad \text { on } \partial \Omega^{\varepsilon}
$$

with $\psi_{j}^{\varepsilon} \in C_{0}^{\infty}\left(\partial \Omega^{\varepsilon}\right), \psi_{j}^{\varepsilon}=o(1)$ in $H^{s-j-1 / 2}, 0 \leqslant j \leqslant s-1$. This can be done with $g_{2}^{\varepsilon} \in C_{(0)}^{\infty}\left(\Omega^{\varepsilon}\right), g_{2}^{\varepsilon}=o(1)$ in $H^{s}(\Omega)$ and the lemma is proved.

We now complete the proof of the theorem. The results of Rauch-Massey [21] imply that the solution of $\tilde{L}^{\varepsilon} u^{\varepsilon}=F^{\varepsilon}, u^{\varepsilon}(0)=g^{\varepsilon}, u^{\varepsilon} \in N^{\varepsilon}$ on $\Gamma^{\varepsilon}$, lies in

$$
\bigcap_{j=0}^{s} C^{j}\left(\bar{I}: H^{s-j}\left(\Omega^{\varepsilon}\right)\right)
$$

As $\varepsilon$ tends to zero the $H^{s}$ norm of $u^{\varepsilon}$ need not stay bounded, however, the tangential estimate (25) shows that $\partial_{t}^{j} u^{\varepsilon}$ is bounded in $C\left(\bar{I}: H_{\tan }^{s-j}\left(\Omega^{\varepsilon}\right)\right)$. Passing to a weak star convergent subsequence yields a solution $u$ to (22) with $\partial_{t}^{j} u \in \mathscr{L}^{\infty}\left(\bar{I}: H_{\tan }^{s-j}(\Omega)\right)$. Estimate (26) applied to the convergent subsequence shows that $\partial_{t}^{j} u \in$ $C\left(\bar{I}: H_{\tan }^{s-j}(\Omega)\right)$ and itself satisfies (25) and (26). By uniqueness this $u$ is the solution.

\section{REFERENCES}

1. R. Agemi, The initial boundary value problem for inviscid harotropic fluid motion, Hokkaido Math. J. 10 (1981), 186-182.

2. C. Bardos and J. Rauch, Maximal positive boundary value problems as limits of singular perturbation problems, Trans. Amer. Math. Soc. 270 (1982), 377-408.

3. D. Ebin, The initial boundary value problem for subsonic fluid motion, Comm. Pure Appl. Math. 32 (1979), 1-19.

4. K. O. Friedrichs, The identity of weak and strong extension of differential operators, Trans. Amer. Math. Soc. 55 (1944), 132-151.

5. Symmetric hyperbolic linear differential equations, Comm. Pure Appl. Math. 7 (1954), $345-392$.

6. Symmetric positive linear differential equations, Comm. Pure Appl. Math. 11 (1958), $333-418$.

7. Choa-Hao Gu, Differentiable solutions of symmetric positive partial equations, Chinese J. Math 5 (1964), 541-545.

8. L. Hormander, Linear partial differential operators, Springer-Verlag, Berlin, 1963.

9. P. D. Lax and R. S. Phillips, Local boundary conditions for dissipative symmetric linear differential operators, Comm. Pure Appl. Math. 13 (1960), 427-454.

10. J. L. Lions and E. Magenes, Non-homogeneous boundary value problems and applications, SpringerVerlag, Berlin and New York, 1972.

11. A. Majda and S. Osher, Initial-boundary value problems for hyperbolic equations with uniformly characteristic boundary, Comm. Pure Appl. Math. 28 (1975), 607-675. 
12. R. Moyer, On the nonidentity of weak and strong extensions of differential operators, Proc. Amer. Math. Soc. 19 (1968), 487-488.

13. T. Nishida and J. Rauch, Local existence for smooth inviscid compressible flows in bounded domains (to appear).

14. S. Osher, An ill-posed problem for a hyperbolic equation near a corner, Bull, Amer. Math. Soc. 79 (1973), 1043-1044.

15. G. Peyser, On the differentiability of solutions of symmetric hyperbolic systems, Proc. Amer. Math. Soc. 14 (1963), 963-969.

16. L. Sarason, Differentiable solutions of symmetrizable and singular svmmetric first order systems, Arch. Rational Mech. Anal. 26 (1967), 357-384.

17. On weak and strong solutions of boundary value problems, Comm. Pure Appl. Math. 15 (1962), 237-288.

18. R. S. Phillips, Dissipative hyperbolic systems, Trans. Amer. Math. Soc. 86 (1957), 109-173.

19. Dissipative operators and hyperbolic systems of partial differential equations, Trans. Amer. Math. Soc. 90 (1959), 249-276.

20. R. S. Phillips and L. Sarason, Singular symmetric positive first order differential operators, J. Math. Mech. 15 (1966), 235-272.

21. J. Rauch and F. Massey, Differentiability of solutions to hyperbolic initial-boundary value problems, Trans. Amer. Math. Soc. 189 (1974), 303-318.

22. S. Schochet, The compressible Euler equations in a bounded domain: Existence of solutions and the incompressible limit, preprint.

23. D. Tartakoff, Regularity of solutions to boundary value problems for first order systems, Indiana Univ. Math. J. 21 (1972), 1113-1129.

24. M. Tsuji, Analyticity of solutions of hyperbolic mixed problems, J. Math. Kyoto Univ. 13 (1973), $323-371$.

Department of Mathematics, University of Michigan, Ann Arbor, Michigan 48109 